\title{
Magnus intersections of one-relator free products with small cancellation conditions
}

\author{
Arye Juhász \\ Dedicated to Professor Avinoam Mann on the occasion of his retirement
}

\begin{abstract}
Donald Collins initiated the study of intersections of Magnus subgroups in onerelator groups. In particular, he characterized those intersections of Magnus subgroups that are not Magnus subgroups. In the present work we show that Collins' results extend to one-relator quotients of free products of groups with a small cancellation condition and give a complete list of those defining relators for which Magnus subgroups do not intersect in a Magnus subgroup. We use van Kampen diagrams and word combinatorics.
\end{abstract}

Mathematics Subject Classification (2000). 20E06,20F05,20F06,20F10.

Keywords. One-relator free products, small cancellation theory, word-combinatorics, Magnus subgroups.

\section{Introduction}

Let $\mathcal{P}=\langle X \mid \mathcal{R}\rangle$ be a presentation of a group. $\mathcal{P}$ is termed one-relator presentation if $\mathcal{R}$ consists of a single relator $R$. We say that a group is a one-relator group if it has a one-relator presentation. Let $G$ be an one-relator group given by the one-relator presentation $\mathcal{P}=\langle X \mid \mathcal{R}\rangle$ and let $F$ be the free group, freely generated by $X$.

The study of one-relator groups started with the pioneering works of Max Dehn and Wilhelm Magnus, and this was one of the central subjects of classical combinatorial group theory (see $[\mathrm{MKS}]$ ). Among the most important achievements of this theory was the solution of the word problem for one-relator groups by W. Magnus. Magnus and his successors developed a whole (algebraic) theory of one-relator groups. The main ingredients of this theory are the subgroups of $G$ which are generated by the images of proper subsets of $X$ under the natural map $\pi: F \rightarrow G$. These groups where termed by his successors Magnus subgroups. Magnus proved that these groups are free, freely generated by the corresponding subset of $\pi(X)$ (the Freiheitssatz). 
Groups in which subgroups generated by proper subsets of a canonical set of generators play a central role, are not exceptional in group theory; for example, if $H$ is a Coxeter group generated by a finite set $S$ of reflections, then the so-called parabolic subgroups - which are the subgroups generated by proper subsets of $S$ enter in considerations of fundamental importance in the theory of Coxeter groups and in representation theory. It is an important basic result that the intersection of two parabolic subgroups is again parabolic.

Coming back to one-relator groups, it is easy to see that intersection of Magnus subgroups is not necessarily a Magnus subgroup: let $X=\{a, b\}$ and let $R=a^{2} b^{3}$. Then $\langle a\rangle$ and $\langle b\rangle$ are Magnus subgroups with non-trivial intersection $\left\langle a^{2}\right\rangle$. However, $\left\langle a^{2}\right\rangle$ is not a Magnus subgroup. This example rises naturally the following questions.

Let $X_{1}$ and $X_{2}$ be proper subsets of $X$, let $Y_{1}=\pi\left(X_{1}\right), Y_{2}=\pi\left(X_{2}\right)$, and let $H_{1}=\left\langle Y_{1}\right\rangle$ and $H_{2}=\left\langle Y_{2}\right\rangle$.

(1) Under what conditions on $R, X_{1}$ and $X_{2}$ is $H_{1} \cap H_{2}$ a Magnus subgroup?

(2) If $H_{1} \cap H_{2}$ is not a Magnus subgroup then how its structure looks like? In particular, how $H_{1} \cap H_{2}$ is related to $\left\langle Y_{1} \cap Y_{2}\right\rangle$ ?

These questions are interesting in their own, but they are also crucial in certain aspects of solutions of equations and also for cyclic presentations. (See [Ju2], [Ju3] and [Ju4], and independently, [E-H].)

The study of Magnus intersections was initiated by Donald Collins in [Co], where among other things he gave a complete answer to the second question, by showing that

$$
\begin{aligned}
& \text { if } H_{1} \cap H_{2} \neq\left\langle Y_{1} \cap Y_{2}\right\rangle \text { then } H_{1} \cap H_{2}=\left\langle Y_{1} \cap Y_{2}\right\rangle *\left\langle c_{1}\right\rangle=\left\langle Y_{1} \cap Y_{2}\right\rangle *\left\langle c_{2}\right\rangle \text {, } \\
& \text { where } c_{1} \in H_{1}, c_{2} \in H_{2} \text { and } c_{1}, c_{2} \notin\left\langle Y_{1} \cap Y_{2}\right\rangle \text {. }
\end{aligned}
$$

Jim Howie in [Ho2], based an a conjecture of Don Collins, gave an algorithm to check whether $H_{1} \cap H_{2}=\left\langle Y_{1} \cap Y_{2}\right\rangle$, or not.

Now, one-relator free products are natural generalisations of one-relator groups: we consider the free group freely generated by $X$ as the free product of infinite cyclic groups and then replace them by arbitrary groups $G_{i}, G_{i} \neq 1$ for $i=1, \ldots, n, n \geq 2$ and take a one-relator quotient (see [Hol] for more motivation). Such groups $G$ have a free product presentations $\mathcal{P}=\left\langle G_{1} * \cdots * G_{n} \mid R\right\rangle$, where $R$ is a cyclically reduced word in $G_{1} * \cdots * G_{n}$ of length at least two. We can naturally extend the notion of Magnus subgroups to one-relator free products, namely a Magnus subgroup of $G$ is a subgroup generated by the image of a proper subset of $\left\{G_{i}\right\}, i=1, \ldots, n$.

In contrast with one-relator groups, very little is known on one-relator free products. Even the most fundamental problem, the word problem, is widely open. Nevertheless, under suitable conditions on the components of the free product or on the defining relator, or on both, large parts of the theory of one-relator groups can be extended to one-relator free products. 
In the present work we make assumptions on $R$ and consider questions 1 and 2 above. More precisely, we assume the small cancellation condition $C(6) \& T(4)$ and in Theorem A, with a mild restriction on $R$ we give a complete classification of those words $R$ for which $H_{1} \cap H_{2}$ is not a Magnus subgroup, where $H_{1}$ and $H_{2}$ are Magnus subgroups of $G$. In Theorem B we show that the corresponding version of the theorem of D. Collins (see [Co]) mentioned above in $(\star)$ holds true. We also show how to get from the defining relator $R$ elements $c_{1}$ and $c_{2}$ in $(\star)$. Finally, in Theorem $C$ we show that Magnus subgroups are free products. (The Freiheitssatz.) We work under the following assumptions

Notation and assumptions of the main theorems. Let $G$ be a group with a onerelator free product presentation $\mathcal{P}, \mathcal{P}=\langle F \mid \mathcal{R}\rangle$, where $F=G_{1} * \cdots * G_{n}, n \geq 2$, $G_{i}, i=1, \ldots, n$, are non-trivial groups, $\mathcal{R}$ is the symmetric closure of a cyclically reduced word $R$ of length at least two in $F$ such that $\mathcal{P}$ satisfies the small cancellation condition $C(6) \& T(4)$. (See [L-S, Ch. V] for definition.) Suppose that no letter in $R$ has order two and if $g \in G_{i}$ occurs in $R$ and $g$ with finite order, then there is at least one more occurrence of a letter in $R$ from $G_{i}$. Let $v: F \rightarrow G$ be the natural homomorphism which sends each element of $F$ to its coset modulo the normal closure of $R$ in $F$. For a subset $Q$ of $\{1, \ldots, n\}$ let $G_{Q}=\underset{i \in Q}{*} G_{i}$. Let $I, J \varsubsetneqq\{1, \ldots, n\}$ such that $I \nsubseteq J$ and $J \nsubseteq I$ and let $D=I \cap J$. Finally, let $H_{Q}$ be the image of $G_{Q}$ by $v$. Our main results are the following.

Theorem A. Let notation and assumptions be as above. If $H_{I} \cap H_{J} \neq H_{D}$ then $R$ has a cyclic conjugate $R^{*}$ which satisfies one of the following:

(i) $R^{*}=U a U^{-1} W^{-1}$ reduced as written, where $a \in G_{i}$ for some $i$ and $(U, W)$ is inadequate (see Definition $2.4(\mathrm{c})$ );

(ii) $R^{*}$ is exceptional in the sense of Definition 5.5;

(iii) $R^{*}=A B$ reduced as written with $A \in G_{I}$ and $B \in G_{J}$.

Moreover, if $R$ has no cyclic conjugate $R^{*}$ as in (i), then $H_{I} \cap H_{J} \neq H_{D}$ if and only if $R^{*}$ is exceptional or $R^{*}=A B, A \in G_{I}$ and $B \in G_{J}$.

The result of Theorem A is quite surprising: clearly, (iii) is an obvious case for $H_{I} \cap H_{J} \neq H_{D}$ and as usual in small cancellation theory we would expect that this is the only case. However, Theorem A tells us that there are also rather unexpected cases (case ii) and moreover, if (i) does not hold then these are all the additional cases. Observe that by Theorem A, the exceptional words in (ii) are precisely those $R$ which have arbitrary length as words in $G_{I} *_{G_{D}} G_{J}$, yet they have a consequence of length two in it.

Theorem B. Let notation and assumptions be as above and suppose that $R$ has no cyclic conjugate $R^{*}$ which satisfies condition (i) of Theorem A. If $H_{I} \cap H_{J} \neq H_{D}$ 
then $H_{I} \cap H_{J}=H_{D} *\langle u\rangle=H_{D} *\langle v\rangle$, where $u \in H_{I} \backslash H_{D}$ and $v \in H_{J} \backslash H_{D}$. Moreover, $R^{*}$ contains a unique subword $U \in G_{I}$ which starts and terminates with a letter in $I \backslash D$ and which is maximal relative to this property and there is a unique subword $V \in G_{J}$ which starts and terminates with a letter in $J \backslash D$ and which is maximal relative to this property such that if $v(U)=u$ and $v(V)=v$ then $H_{D} *\langle u\rangle=H_{D} *\langle v\rangle=H_{I} \cap H_{J}$.

Theorem C. Let notation and assumptions be as above and suppose that no cyclic conjugate $R^{*}$ of $R$ satisfies condition (i) of Theorem $A$. Then $H_{J} \cong G_{J}$. In particular, $H_{J}$ is a free product.

We mention that J. Howie in [Ho2], independently, considered problems (1) and (2) above in one-relator free products with arbitrary defining relators, however, with the assumptions that every component $G_{i}$ is locally indicable (i.e. every finitely generated non-trivial subgroup maps onto the infinite cyclic group).

Our main tools are small cancellation theory and van Kampen diagrams with word combinatorics. We prove first Theorem C. The idea is to show that under the assumptions of the theorem,

every consequence of the defining relator $R$ contains at least one letter from each $G_{i}$.

(This is one of the equivalent formulations of the Freiheitssatz by Magnus. See [L-S].)

A central ingredient in small cancellation theory is Greendlinger's Lemma, which guaranties the existence of at least two Greendlinger regions in every van Kampen diagram $M$, which has at least two regions. These are regions with the property that their boundary has a large common portion with the boundary of $M$. (For definitions of van Kampen diagrams and regions see Section 2.2.)

However for our problem, showing $(\star \star)$, a more precise information than just knowing that a large portion of the defining relator is present on the boundary of $M$, is needed.

Recently we developed an improved version of Greendlinger's Lemma for onerelator groups and one-relator free products with the small cancellation condition $C(6) \& T(4)$, which implies $(\star \star)$, and hence proves Theorem C. We remark that it also implies several results of different nature. In [Ju5] we solved the membership problem for Magnus subgroups of one-relator free products with small cancellation. In [Ju6] we proved the appropriate version of Magnus's Freiheitssatz for Magnus subsemigroups of one-relator groups with small cancellation. In [Ju7] we classify non-malnormal Magnus subgroups in one-relator groups and free products with small cancellation. We also plan to use it in complexes of certain types of groups to produce a lower bound on the angles between the local groups.

Theorem B follows easily from the proof of Theorem A, so we concentrate on the proof of Theorem A. The proof of Theorem A is much more demanding then the 
proof of Theorem C; while the improved Greendlinger's Lemma was enough for the proof of Theorem $\mathrm{C}$, we need the extension of a further result from small cancellation theory. If $H_{I} \cap H_{J} \neq H_{D}$, as in Theorem $\mathrm{A}$, then there are non-empty words $A$ and $B$ in $G_{I}$ and $G_{J}$ respectively, such that $v(A)=v(B)$ in $G$. Thus $A B^{-1}$ is a consequence of $R$ and hence there is a van Kampen diagram $M$ with boundary label $A B^{-1}$. In Theorem A we recover the combinatorial structure of the word $R$ from the combinatorial structure of its consequence $A B^{-1}$. In a sense, we deal with an inverse problem to the word problem. In the word problem we are given $R$ and we want to check whether (another) given word $W$ is a consequence of $R$; in Theorem $\mathrm{A}$ a word $W\left(=A B^{-1}\right)$ is given and it is also given that $W$ is a consequence of an unknown relation $R$, and we would like to find the combinatorial structure of all such relations, in term of the combinatorial structure of $W$. We are not aware of results in this direction in the literature. This is a difficult problem in general, because boundary regions of $M$ contribute only parts of $R^{*}$ to the boundary label of $M$ and in general, it is difficult to recover $R^{*}$ from these parts. There is however, one case when this is doable; this is the case when $M$ is a one-layer diagram. Then we can use word combinatorics in order to determine the combinatorial structure of $R$. This is done in Sections 5.1 and 5.2. So it remains now to show that $M$ is a one-layer diagram. We show this in Section 4.

The work is organised as follows:

In Section 2 we introduce preliminary results on words and van Kampen diagrams as well as the improved version of Greendlinger's Lemma. In Section 3 we prove Theorem $\mathrm{C}$ while in Section 4 we prove that intersection diagrams are one-layer diagrams. In Section 5 we prove Theorems A and B.

Acknowledgements. I am grateful to Don Collins for showing me and discussing with me results from [Co] prior to publication. I am also grateful to the referee of a previous version for his useful remarks which improved the presentation of the work. My thanks go to Zoran Šunić and the editors of this issue for their help in bringing this paper to publication.

\section{Preliminary results on words and diagrams}

2.1. Words. Basic reference for this subsection is [L-S, Ch. V]. We recall here only a few basic notions and results which we need.

Let $F=G_{1} * \cdots * G_{n}, n \geq 2$, be the free product of non-trivial groups $G_{i}$, $i=1, \ldots, n$. We call the $G_{i}$ s the components of $F$. Let $G$ be a group. A free presentation for $G$ is a presentation of $G$ as a homomorphic image of free group $F$. A free product presentation for $G$ is a presentation of $G$ as a homomorphic image of free product $F$. If $F$ is a free group, freely generated by a set $X$ then, as usual, we denote free presentation of $G$ by $\langle X \mid \mathcal{R}\rangle$, where $\mathcal{R}$ is a set of defining relations 
for $G$, and if $F$ is a free product, $F=G_{1} * \cdots * G_{n}, n \geq 2, G_{i}$ non-trivial, then we denote free product presentation of $G$ by $\left\langle G_{1} * \cdots * G_{n} \mid \mathcal{R}\right\rangle$, where again $\mathcal{R}$ is a set of defining relations for $G, \mathcal{R} \subseteq G_{1} * \cdots * G_{n}$. The elements of $F \backslash\{1\}$ can be uniquely presented by finite sequences of non-trivial elements of the components, such that adjacent elements in a sequence come from different components. We call the elements of $G_{i}, i=1, \ldots, n$, letters and the sequences of elements, words. For $g \in G_{i}, g \neq 1$, denote $\alpha(g)=i$. Thus, if $1 \neq W \in F$ then $W$ can be uniquely expressed as a word: $W=b_{i_{1}} \ldots b_{i_{k}}$, where $k \geq 1,1 \neq b_{i_{j}} \in G_{i_{j}}$ and $\alpha\left(b_{i_{j}}\right) \neq \alpha\left(b_{i_{j+1}}\right)$ for $j=1, \ldots, k-1$. We call this presentation of $W$ its normal form, call $k$ its length and denote it by $|W|$.

Let $U$ and $V$ be reduced words in $F$. We say that the product $U V$ is reduced as written if either the last letter of $U$ and the first letter of $V$ are in different components $G_{i}$, or if there is no cancellation between $U$ and $V$, however the last letter of $U$ and the first letter of $V$ may come from the same component (consolidation).

Denote by $\mathscr{H}(W)$ the set of initial subwords of $W$ and by $\mathcal{T}(W)$ the set of terminal subwords of $W$. Also, for a reduced non-empty word $W$ we denote by $h(W)$ the first letter of $W$ and by $t(W)$ the last letter of $W$. We start with the following well-known results on word equations over $F$.

Lemma 2.1. (a) Let $A, B$ and $C$ be reduced words which contains no letters of order two, such that $A B$ and $B C$ are reduced as written. If $|A B| \geq 2$ and $A B=B C$ then $A=K L, C=L K$ and $B=(K L)^{\beta} K, \beta \geq 0$.

(b) Let $A$ be a cyclically reduced word, $|A| \geq 2$. If $A A=U A^{\varepsilon} V$, reduced as written, $U \neq 1, V \neq 1$, and $\varepsilon \in\{1,-1\}$, then $\varepsilon=1$ and $A=B^{k}, k \geq 2$, for some cyclically reduced word $B$.

(c) Let $Z$ be a reduced word which contains no letters of order two.

(i) If for some reduced words $V$ and $U$, such that $Z U$ and $Z^{-1} V$ are reduced as written, we have $Z U=Z^{-1} V$, then $|Z|=1$ and $V=Z^{2} U$. Moreover, $Z$ and the first letters of $V$ and $U$ are in the same component $G_{i}$.

(ii) If for some reduced words $U$ and $V$ such that $U Z$ and $Z^{-1} V$ are reduced as written, we have $U Z=Z^{-1} V$, then $U=Z^{-1} a$ and $V=a Z$, where $a$ is $a$ letter and the first letter of $Z$ and $a$ are in the same component $G_{i}$.

We introduce below the key notion of the work.

Definitions and notation. (a) Let $W \in F, W=a_{i_{1}} \ldots a_{i_{k}}, a_{i_{j}} \in G_{i_{j}}$ reduced as written. Define

$$
\operatorname{Supp}(W)=\left\{i_{1}, \ldots, i_{k}\right\} \subseteq\{1, \ldots, n\} .
$$

(b) Let $W_{1}$ and $W_{2}$ be reduced words in $F$. $W_{2}$ majorises $W_{1}$ if $\operatorname{Supp}\left(W_{2}\right) \supseteq$ $\operatorname{Supp}\left(W_{1}\right)$. In this case write $W_{2} \succ W_{1}$. If $W_{1} \succ W_{2}$ and $W_{1} \succ W_{3}$ we shall write $W_{1} \succ W_{2}, W_{3}$. 
(c) For $W_{1}$ and $W_{2}$ in part (b) define $W_{1} \sim W_{2}$ if $W_{1} \prec W_{2}$ and $W_{2} \prec W_{1}$. Thus $W_{1} \sim W_{2}$ if and only if $\operatorname{Supp}\left(W_{1}\right)=\operatorname{Supp}\left(W_{2}\right)$.

Clearly " $\sim$ " is an equivalence relation, which contains the equality of elements in $F$.

The following lemma is immediate from the definition, hence its proof is omitted.

Lemma 2.2. (a) If $A$ is a subword of $B$ then $A \prec B$.

(b) If $A \prec B$ then $A^{ \pm 1} \prec B^{ \pm 1}$.

(c) If $A \sim B$ and $A \prec C$ then $B \prec C$.

(d) If $A=P_{1} \ldots P_{m}$, reduced as written and $P_{i} \sim Q$ for $i=1, \ldots, m$ and $a$ reduced word $Q$, then $A \sim Q$.

(e) If $A \succ P_{1}, \ldots, P_{m}$ then $A \succ W\left(P_{1}, \ldots, P_{m}\right)$, for every word $W$ on $P_{1}, \ldots, P_{m}$.

Parts (a) and (b) of the following lemma are immediate corollaries of Lemma 2.1 and Lemma 2.2. Also, the remaining parts are routine case by case checking. Hence we omit their proofs.

Lemma 2.3. (a) Let $A, B$ and $C$ be as in Lemma 2.1 (a). Then $B \prec A \sim C \sim A B \sim$ $B C$. If $\beta \geq 1$ then $B \sim A$.

(b) If $A B=K A C$ with $|B| \geq 2$ and $|K A| \geq 2$, reduced as written then $B \succ A, B \succ C$ and $K \succ A$.

(c) Let $K, Q, U, V$ and $S$ be non-empty words such that $K Q, U V, V U$ and $K S$ are reduced as written, of length at least two. If $K Q=U V$ and $K S=V U$ then either $Q \sim S \succ K, U, V$, or $U=D^{\alpha}, V=D^{\beta}, \alpha, \beta \geq 1$ and $D \succ K, Q, S$.

(d) Let $B, Q, L, U$ and $V$ be non-empty words such that $B Q, U V, L B$ and $V U$ have length at least two and are reduced as written. If $B Q=U V$ and $L B=V U$ then one of the following holds:

(i) $B=U, Q=L=V$; or

(ii) $Q \succ B, U, V, L$ and $L \succ B, U, V, Q$ (hence $L \sim Q \sim U V$ ).

(e) Let $L, K, Q_{1}, M$ and $N$ be non-empty reduced words, such that $K Q_{1}, M N, Q_{1} M$ and $L K$ are reduced as written with length at least two. If $K Q_{1}=M N$ and $Q_{1} M=L K$, then one of the following holds:

(i) $Q_{1}=N=L$ and $K=M$; or

(ii) $Q_{1} \succ K, L, M, N$.

Notice that if one of the products in parts (a)-(e), like $B Q$ in part (d), has length one then the statements of Lemma 2.3 trivially hold true.

The following basic notions are crucial for the paper. 
Definition 2.4. (a) Let $R$ be a weakly cyclically reduced word in $F$ and let $P$ be a subword of a cyclic conjugate of $R . P$ is a piece in $R$ (or a piece relative to the symmetric closure $\mathcal{R}$ of $R$ ) if $R$ has distinct cyclic conjugates $R_{1}$ and $R_{2}$ such that $R_{1}=P R_{1}^{\prime}, R_{2}^{\varepsilon}=P R_{2}^{\prime}$, reduced as written, for some $\varepsilon \in\{1,-1\}$. Equivalently, $P^{ \pm 1}$ has at least two occurrences in the cyclic word $\hat{R}$, corresponding to the linear word $R$. We call the two occurrences of $P$ in $R_{1}$ and $R_{2}^{\varepsilon}$, respectively, a piece pair and denote it by $\left(P, P^{\prime}\right)$, where $P^{\prime}\left(=P^{\varepsilon}\right)$ is the occurrence of $P^{\varepsilon}$ in $R_{2}$.

(b) A piece pair $\left(P, P^{\prime}\right)$ as in part (a) of the definition is right normalized if $\left(R_{1}^{\prime}\right)^{-1} R_{2}^{\prime}$ is reduced as written.

(c) Let $R=U a U^{-1} W^{-1}$, reduced as written, $a \in G_{i}$ for some $i, i=1, \ldots, n$. The pair $(U, W)$ is inadequate if

(i) $W$ is the product of at least four pieces over the symmetric closure of $R$ and

(ii) $\operatorname{Supp} W \supsetneqq \operatorname{Supp} U$.

2.2. Diagrams. For basic results on diagrams see $[\mathrm{L}-\mathrm{S}, \mathrm{Ch}$. V]. We recall here some of the basic definitions from [L-S, p. 236 and pp. 274-276] for convenience.

A diagram over a group $F$ is an oriented map $M$ and a function $\Phi$ assigning to each oriented edge $e$ of $M$ as a label an element $\Phi(e)$ of $F$ such that if $e$ is an oriented edge of $M$ and $e^{-1}$ the oppositely oriented edge, then $\Phi\left(e^{-1}\right)=\Phi(e)^{-1}$, and if $\mu=e_{1} v_{1} e_{2} v_{2} \ldots e_{k}$ is a path in $M$ then $\Phi(\mu)=\Phi\left(e_{1}\right) \Phi\left(e_{2}\right) \ldots \Phi\left(e_{k}\right)$. We denote by $\Phi_{M}$ the labelling function of $M$ over $F$. If $M$ is fixed we shall write $\Phi$ for $\Phi_{M}$.

If $M$ is planar, connected and simply connected then it is called $a$ van Kampen diagram. In the case of diagrams $M$ over free products the vertices are divided into two types, primary and secondary. The label on every edge of $M$ will belong to a factor $G_{i}$ of $F$ with the labels on successive edges meeting at primary vertices belonging to different factors $G_{j}$, while the labels on the edges at a secondary vertex all belong to the same factor of $F$. For a region $D$ in $M$ denote by $\partial D$ its boundary and by $\partial M$ the boundary of $M$.

Definitions 2.5. Let $M$ be a diagram over $F$.

(a) Two regions $D_{1}$ and $D_{2}$ in $M$ are neighbours if $\partial D_{1} \cap \partial D_{2} \neq \emptyset$. They are proper neighbours if $\partial D_{1} \cap \partial D_{2}$ contains a non-empty edge.

(b) A region $D$ is a boundary region if $\partial D \cap \partial M \neq \emptyset$. A region $D$ is a proper boundary region if $\partial D \cap \partial M$ contains a non-empty edge. A region of $M$ which is not a boundary region is an inner region.

(c) Let $M$ be a connected, simply connected map. $M$ is a simple one-layer map, if the dual map $M^{*}$, obtained from $M$ by putting in each region $D$ a vertex $D^{*}$ and connecting two vertices $D_{1}^{*}$ and $D_{2}^{*}$ by an edge if $D_{1}$ and $D_{2}$ are proper neighbours, is a tree in which each vertex has valency at most two. (See 
Figure 1 (b).) In particular, $M$ has connected interior, every region is a boundary region, each region has at most two proper neighbours and if $M$ contains more than one region then $M$ contains exactly two regions, see $D_{1}, D_{r}$ in Figure 1 (b) and $D_{1}, D_{2}$ in Figure 1 (c), which have exactly one neighbour each. $M$ is a one-layer map if it is composed from simple one-layer maps and paths in the way shown in Figure 1 (a).

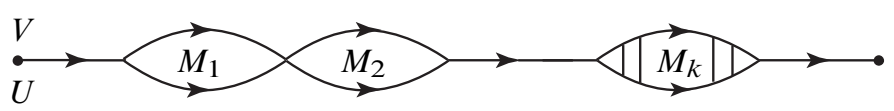

(a)

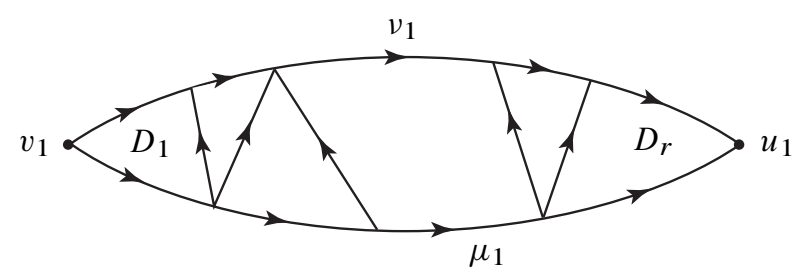

(b)

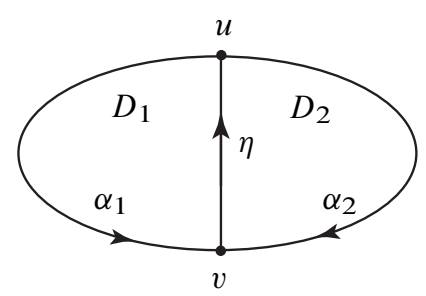

(c)

Figure 1. One-layer diagrams.

We shall need the next lemma in Section 5. As pointed out by the referee it is an immediate consequence of Lemma 2.1. We omit its proof.

Lemma 2.6. Let $\mathcal{R}$ be the symmetric closure of a cyclically reduced word $R$ and let $M$ be a van Kampen diagram over $\mathcal{R}$, with a boundary label K. Let $D_{1}$ and $D_{2}$ be adjacent regions in $M$ with boundary cycles $u \alpha_{1} v \eta u$ and $u \eta^{-1} v \alpha_{2}^{-1} u$, respectively, where $u$ and $v$ are vertices. (See Figure 1 (c).) Suppose $R$ has a cyclic conjugate $A^{n}$, $n \geq 2$ for some cyclically reduced non-empty word A. Suppose further that

(i) $A$ is not a proper power (i.e. $A \neq B^{k}, k \geq 2$ for every word $B$ ). 
(ii) $M$ contains a minimal number of regions among all the diagrams with boundary label $K$.

Then $\Phi(\eta)$ contains no cyclic conjugate of $A^{ \pm 1}$.

We recall the main structure theorem from [Ju1], where it is proved in a more general setting. Observe that the condition $C(6) \& T(4)$ implies the condition $W(6)$ in [Ju1]. (For the definition of the standard small cancellation conditions, see [L-S, pp. 240-241]

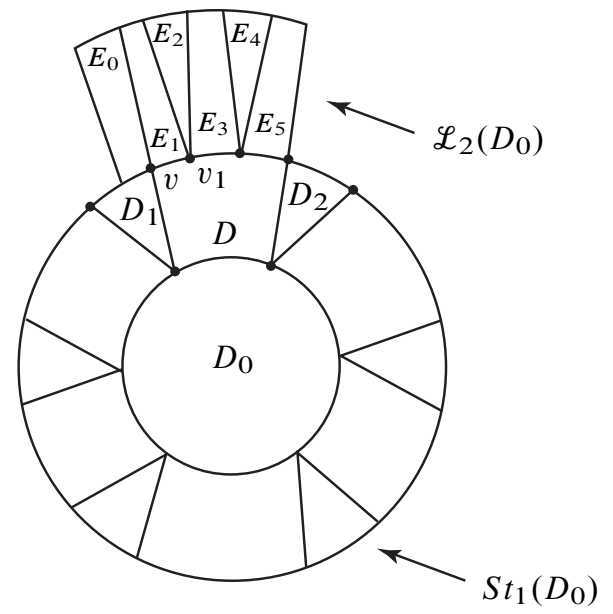

(a)

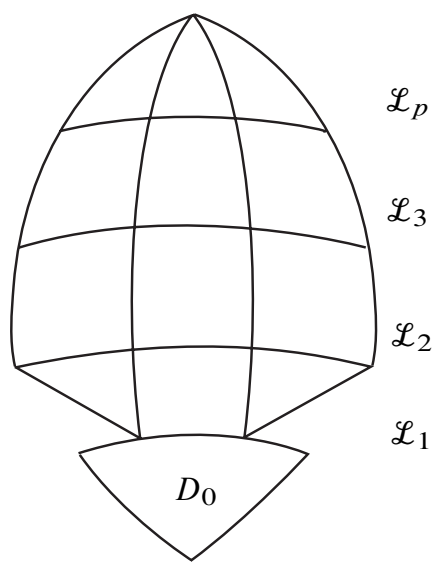

(b)

Figure 2. Layer decompositions.

Theorem 2.7 (Layer decomposition, [Ju1]). (See Figure 2.) Let $M$ be a simply connected map (diagram) with connected interior and let $D_{0}$ be a region of $M$. Assume that $M$ satisfies the condition $C(6) \& T(4)$.

Define $S t_{0}\left(D_{0}\right)=D_{0}$ and for $i \geq 1$ let $S t_{i}\left(D_{0}\right)=S t_{i-1}(D) \cup \mathscr{L}_{i}\left(D_{0}\right)$, where $\mathscr{L}_{i}\left(D_{0}\right)=\left\langle D\right.$ in $\left.M \backslash S t_{i-1}\left(D_{0}\right) \mid \partial D \cap \partial S t_{i-1}\left(D_{0}\right) \neq \emptyset\right\rangle$ and $\mathscr{L}_{0}=\left\{D_{0}\right\}$. Let $p$ be the smallest number such that $S t_{p}\left(D_{0}\right)=M$ and assume that $p>0$ (i.e., $M$ contains more than one region). Then each of the following holds:

(a) Every regular submap of $S t_{i+1}\left(D_{0}\right)$ containing $S t_{i}\left(D_{0}\right)$ is simply connected for $0 \leq i \leq p$. (A submap is regular if every edge is on the boundary of a region.)

(b) Every connected and simply connected submap of $\mathscr{L}_{i}\left(D_{0}\right)$ is a one-layer map.

(When $D_{0}$ is fixed, we shall abbreviate $\mathscr{L}_{i}\left(D_{0}\right)$ by $\mathscr{L}_{i}$ and call $\Lambda\left(D_{0}\right)=\left(\mathscr{L}_{0}, \ldots, \mathscr{L}_{p}\right)$ a layer decomposition of $M$. We call $D_{0}$ the center of the layer decomposition.) 
(c) For a region $D \in \mathscr{L}_{i}, i \geq 1$ denote by $\mathcal{A}(D)$ the set of regions $E$ in $\mathscr{L}_{i-1}$, which have a non-trivial common edge with $D$, denote by $\mathscr{B}(D)$ the set of regions $S$ in $\mathscr{L}_{i}$ with $\partial S \cap \partial D \neq \varnothing$ and denote by $\mathcal{C}(D)$ the set of regions $K$ of $\mathscr{L}_{i+1}$, $(i<p)$ with $\partial K \cap \partial D \neq \emptyset$. Also, let $a(D)=|\mathcal{A}(D)|, b(D)=|\mathscr{B}(D)|$ and $c(D)=|\mathcal{C}(D)|$. Then $a(D) \leq 1$ and $b(D) \leq 2$. In other words, $D$ has at most two proper neighbours in $\mathscr{L}_{i}$ and at most one neighbour in $\mathscr{L}_{i-1}$.

(d) If $v \in \partial S t_{i}\left(D_{0}\right) \backslash \partial S t_{i-1}\left(D_{0}\right)$ then $v$ has valency at most three in $S t_{i}\left(D_{0}\right)$.

(e) For regions $D, E$ in $M$ with $\partial D \cap \partial E \neq \emptyset$ we have that $\partial D \cap \partial E$ is connected.

Remark. Let $M$ be a connected, simply connected map (diagram) with connected interior and let $D$ be a region in $M$. Let $\Lambda(D)$ be a layer decomposition of $M$ with center $D$. Suppose that $D$ is a boundary region of $M$ with a non-empty edge on $\partial M$. (See Figure 3.) Then it follows from the above theorem that $\mathscr{L}_{1}(D)$ is not annular, hence simply connected, though not necessarily with connected interior. (See Figure 3 (a), where the interior of $\mathscr{L}_{1}$ is simply connected and connected and see Figure $3(\mathrm{~b})$, where the interior of $\mathscr{L}_{1}$ is not connected.) But then due to the simply connectedness of $M, \mathscr{L}_{i}$ is simply connected for every $i$.

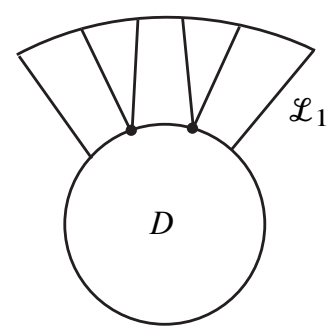

(a)

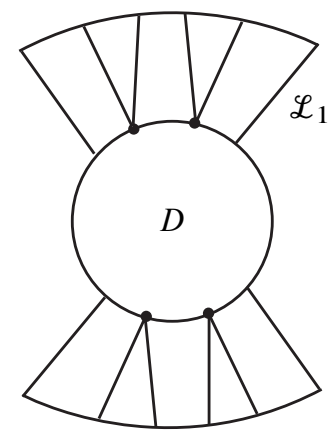

(b)

Figure 3. Simply connected and not simply connected layers.

In the next definition we introduce special subdiagrams and regions, the boundaries of which share a large portion with the boundary of $M$.

Definition 2.8. (a) Let $\Lambda\left(D_{0}\right)$ be a layer decomposition of $M$, where $D_{0}$ is a boundary region of $M$ with a non-empty edge on $\partial M$. A connected component $P$ of the interior of $\mathscr{L}_{i}$ is a peak relative to $D_{0}$, if either $i=p$ or no region of $\mathscr{L}_{i+1}$ is a neighbour of any region in $P$. If $\Lambda\left(D_{0}\right)=\left(\mathscr{L}_{0}, \mathscr{L}_{1}, \ldots, \mathscr{L}_{p}\right)$ then the closure of every connected 
component $P$ of the interior of $\mathscr{L}_{p}$ is a peak. (See Figure $2\left(\right.$ a), where $p=2$ and $\mathscr{L}_{2}$ is a peak and Figure 2 (b), where $\mathscr{L}_{p}$ is a peak.) Related to peaks is the following notion.

(b) A boundary region $D$ of $M$ is a $k$-corner region for $k=1,2$ if each of the following holds:

1) $\partial D \cap \partial M$ is connected and

2) $D$ has $k$ proper neighbours in $M$.

Example 2.9. Let $M$ be a diagram of a $C(6) \& T(4)$ presentation. Let $P$ be a peak, depicted in Figure 4 (a). Then its extremal regions $D_{1}$ and $D_{k}$ are 2-corner regions because $a\left(D_{1}\right) \leq 1$ and $b\left(D_{1}\right) \leq 1$, due to being extremal. If $P$ is a peak consisting of a single region $E$, then $E$ is a 1-corner region due to Theorem 2.7. Also, if $a\left(D_{k-1}\right)=0$ then $D_{k-1}$ in Figure $4($ a) is a 2-corner region.

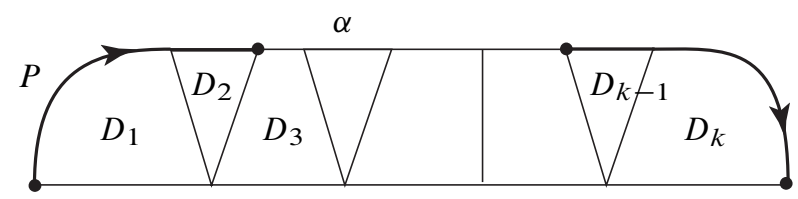

(a)

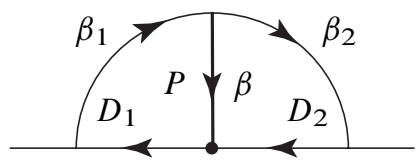

(b)

Figure 4. Peaks and corner regions in van Kampen diagrams.

The $k$-corner regions are examples of Greendlinger regions. (These are regions which satisfy the conditions of Greendlinger's Lemma. See [L-S, pp. 250-251].)

The next section is devoted to the improved version of Greendlinger's Lemma. A similar version was formulated in [Ju5] the proof of which, using Lemmas 2.3 and 2.11, easily can be adapted to the proof of Proposition 2.12 below. Therefore, we shall omit the details of the proof, which consists of case by case checking.

2.3. An improved version of Greendlinger's Lemma. The improved version of Greendlinger's Lemma is given for 1-corner regions in Lemma 2.10 and for 2-corner regions in Proposition 2.12. 
In this section we assume that the conditions of Theorem A are satisfied.

2.3.1. 1-corner regions. Let $D$ be a 1-corner region in $M$ with proper neighbour $E$. Let $\alpha=\partial D \cap \partial E$, let $P=\Phi(\alpha)$, let $\eta=\partial D \cap \partial M$ and let $Q=\Phi(\eta)$. (See

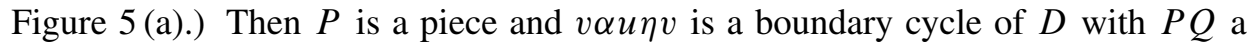
boundary label of $D$, where $u$ and $v$ are vertices.

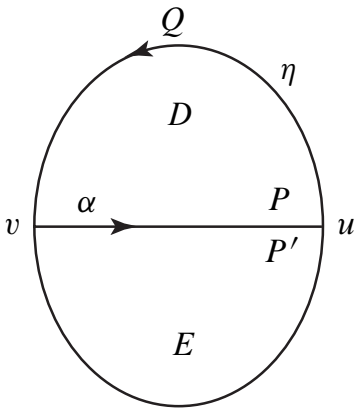

(a)

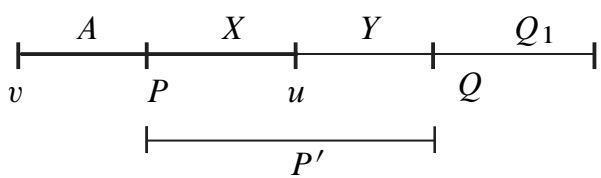

(b)

Figure 5. 1-corner regions and corresponding word equation.

Lemma 2.10. Let notation be as above. Then $Q \succ P$.

Proof. Let $\left(P, P^{\prime}\right)$ be the corresponding piece pair. Then one of the following holds:

1) $P^{\prime}$ is a subword of $Q$;

2) $P^{\prime}$ overlaps with $P$.

In case 1) $Q \succ P$, by Lemma 2.2 (a). Also, in case 2), if $|P|=1$ then $Q \succ P$. Hence assume that $|P| \geq 2$. In case 2) we have $P=A X, P^{\prime}=X Y, Q=Y Q_{1}$, reduced as written, $Q_{1} \in \mathcal{T}(Q)$. See Figure 5(b). Applying Lemma 2.3 (a) to the first two of these equations and remembering that $P^{-1}$ cannot overlap $P$ in more than one letter (see Lemma 2.1 (c)), we get $A \sim Y \succ X$ and hence, by Lemma 2.2, $P \sim Y$. Applying Lemma 2.2 to the last equation implies $Q \succ P$.

The lemma is proved. 
2.3.2. 2-corner regions. Let $D$ be a 2-corner region in $M$ with neighbours $E_{r}$ and $E_{\ell}$. See Figure 6. Denote $\alpha_{1}=\partial D \cap \partial E_{r}$ and denote $\alpha_{2}=\partial D \cap \partial E_{\ell}$. Let $v_{0}=\alpha_{1} \cap \partial M$, let $v_{2}=\alpha_{2} \cap \partial M$ and let $v_{1}=\alpha_{1} \cap \alpha_{2}$. Denote $P_{1}=\Phi\left(\alpha_{1}\right)$, $P_{2}=\Phi\left(\alpha_{2}\right)$ and $Q=\Phi(\partial D \cap \partial M)$. Let $\left(P_{1}, P_{1}^{\prime}\right)$ and $\left(P_{2}, P_{2}^{\prime}\right)$ be the piece pairs obtained from $\alpha_{1}$ and $\alpha_{2}$, being common edges of $\partial E_{\ell}$ and $\partial D$ and of $\partial E_{r}$ and $\partial D$, respectively. Thus, $P_{1}^{\prime}$ and $P_{2}^{\prime}$ are subwords of $\Phi\left(\partial E_{r}\right)$ and $\Phi\left(\partial E_{\ell}\right)$, respectively, which are equal to $P_{1}$ and $P_{2}$, respectively, as words, and since all the regions of $M$ have the same boundary labels, up to sign, $\left(P_{1}, P_{1}^{\prime}\right)$ and $\left(P_{2}, P_{2}^{\prime}\right)$ are piece pairs. It is convenient and harmless to identify $P_{i}$ with $\alpha_{i}$ and, similarly, $P_{i}^{\prime}$ with $\alpha_{i}^{\prime}, i=1,2$. Then $v_{2} Q v_{0} P_{1} v_{1} P_{2} v_{2}$ is a boundary label of $D$, which we may assume to coincide with $R$, without loss of generality, where $P_{1}$ and $P_{2}$ are pieces.

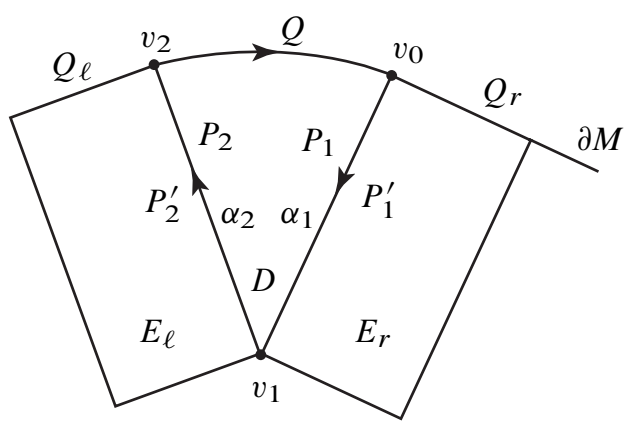

Figure 6. 2-corner regions.

The proof of the following lemma is a routine case by case checking, hence we omit it.

Lemma 2.11. Let notation be as above and make the assumptions of the main theorems. Assume that condition (i) of Theorem A is not satisfied by any cyclic conjugate of $R$.

(a) If $\left|P_{1}\right|=1, v_{0}$ has valency three in $M$ and $v_{1}$ is an inner vertex of $M$, then $Q \succ P_{1}, P_{2}$. Similarly, if $\left|P_{2}\right|=1$ and $v_{1}$ is an inner vertex of $M$, then $Q \succ P_{1}, P_{2}$.

(b) Suppose $\left|P_{1}\right| \geq 2$ and $P_{1}$ overlaps with $P_{1}^{\prime}$. If $\varepsilon_{1}=-1$ then either $P_{1} \prec Q$ or $P_{1} \prec Z Q$, where $P_{2}=c P_{1}^{-1} Z$ and where $c$ is a letter with $\alpha(c)=\alpha\left(h\left(P_{1}^{-1}\right)\right)$ and $P_{1}^{\prime}$ contains $v_{1}$. The analogous result holds for $P_{2}$.

The following is our version to Greendlinger's Lemma for 2-corner regions. 
Proposition 2.12. Let notation be as above and assume that $R$ satisfies the assumptions of the main theorems, and no cyclic conjugate of $R$ satisfies condition (i) of Theorem A. Assume that the piece pairs $\left(P_{1}, P_{1}^{\prime}\right)$ and $\left(P_{2}, P_{2}^{\prime}\right)$ are right normalised. Let $Q_{r}=\partial E_{r} \cap \partial M$ and let $Q_{\ell}=\partial E_{\ell} \cap \partial M$. Then the following holds:

If $d_{M}\left(v_{0}\right)=3$ and $Q_{r}$ is not a piece, then $Q_{r}$ has a head $Q_{\rho}$ which is a piece over $\mathcal{R}$ such that $Q Q_{\rho} \succ P_{1} P_{2}$ and dually, if $d_{M}\left(v_{2}\right)=3$ and $Q_{\ell}$ is not a piece, then $Q_{\ell}$ has a tail $Q_{\lambda}$ which is a piece over $\mathcal{R}$ such that $Q_{\lambda} Q \succ P_{1} P_{2}$. In particular, if both $v_{0}$ and $v_{2}$ have valency three and both $Q_{r}$ and $Q_{\ell}$ are not pieces (i.e. the products of at least two pieces) then both $Q_{\lambda} Q \succ R$ and $Q Q_{\rho} \succ R$ hold true.

We close this section with the following consequence of the proposition.

Proposition 2.13. Let $M$ be an $\mathcal{R}$-diagram. Let assumptions be as in Proposition 2.12. Let $P$ be a peak relative to a layer decomposition $\Lambda$. Let $\alpha=\partial P \cap \partial M$. Then $\Phi(\alpha)$ contains a letter from each component.

Proof. Let $P=\left\langle D_{1}, \ldots, D_{k}\right\rangle$. If $k=1$ then the result follows from Lemma 2.10. If $k \geq 3$ then it follows from Theorem 2.7 (d) and the $T(4)$ condition that either $P$ contains a 1-corner region or contains a 2-corner region $D$ with two neighbours $E_{r}$ and $E_{\ell}$ such that $\partial D \cap \partial E_{r} \cap \partial M$ and $\partial D \cap \partial E_{\ell} \cap \partial M$ are vertices with valency three and $\partial E_{r} \cap \partial M$ and $\partial E_{\ell} \cap \partial M$ are not pieces (due to the $C(6)$ condition). In both cases the result follows by Proposition 2.12, where $D_{1}$ is $E_{\ell}, D_{2}$ is $D$ and $D_{3}$ is $E_{r}$. See Figure 4 (a).

Finally, assume $k=2$. See Figure 4 (b). Let $P=\left\langle D_{1}, D_{2}\right\rangle$, let $\beta_{1}=\partial D_{1} \cap \partial M$ and let $\beta_{2}=\partial D_{2} \cap \partial M$. Both $D_{1}$ and $D_{2}$ are 2-corner regions, and $\beta_{i}$ is the product of at least four $(4=6-2)$ pieces, for $i=1,2$. Also, by Theorem 2.7 (d), $\beta_{1} \cap \beta_{2}$ is a vertex with valency three. Therefore, by Proposition 2.12, $\beta_{1} \cup \beta_{2}$ contains a letter from each component $G_{j}$ and the proposition is proved.

\section{The proof of Theorem $\mathrm{C}$}

In proving Theorem $\mathrm{C}$ we may assume without loss of generality that $\operatorname{Supp}(R)=$ $I \cup J=\{1,2, \ldots, n\}$ and we shall do so.

Suppose $H_{J}$ is not a free product. Then there exists a non-empty word $W$ in $G_{J}$ such that $v(W)=1$ in $G$. Therefore, by [L-S, Theorem 1.1, p. 237] there exists a connected, simply connected diagram $M$ with boundary label $W$. Let $\Delta$ be a connected component of the interior of $M$. By Proposition $2.13 \partial \Delta$ contains a letter from every component $G_{i}$ for $i=1, \ldots, n$. Since, as sets, $\partial \Delta \subseteq \partial M$, $\partial M$ also contains a letter from every component. This, however, violates $W \in G_{J}$, $J \varsubsetneqq\{1, \ldots, n\}$. Therefore, $H_{J}$ is a free product, $H_{J} \cong G_{J}$.

The theorem is proved. 


\section{The structure of intersection diagrams}

For the proof of Theorems A and B we may assume without loss of generality that $\operatorname{Supp}(R)=I \cup J=\{1, \ldots, n\}$ and we shall do so. In this section we shall assume the notation of the main theorems, and, moreover, that $R$ has no cyclic conjugate $R^{*}$ which satisfies condition (i) of Theorem A. Let $W \neq 1$ be an element of $H_{I} \cap H_{J}$. Then there are non-empty words $U$ in $G_{I}$ and $V$ in $G_{J}$ such that $v(U)=v(V)$ in $G$. Hence, by [L-S, Theorem 1.1, p. 237] there is a van Kampen $\mathcal{R}$-diagram $M$ with boundary label $U V^{-1}$. We call this diagram an Intersection Diagram.

Definition 4.1. Let $D=I \cap J$ and let $F_{I, J}=\left\langle G_{I \cup J}\right\rangle$. We can consider $F_{I, J}$ as the amalgamated free product $F_{I, J}:=G_{I} *_{G_{D}} G_{J}$. We shall denote the length of a word $W$ in $F$, considered as a word in $F_{I, J}$ by $\|W\|$.

Proposition 4.2. Let assumptions be as above. Let $M$ be an intersection diagram with boundary label $U V^{-1}$, where $U \in G_{I}$ and $V \in G_{J}$. If $\|R\| \geq 4$ and $\left\|U V^{-1}\right\|=2$ then $M$ is a one-layer diagram.

We need Lemmas 4.4 and 4.5 for the proof of Proposition 4.2. In what follows we shall use the notation and rely on the assumptions of Proposition 4.2. Also, we shall use the following easy lemma, the proof of which we omit.

Lemma 4.3. Let $\alpha_{1}, \alpha_{2}$ and $\alpha_{3}$ be disjoint boundary paths of $M$. Let $\omega$ be a boundary cycle of $M$. Then $\|\Phi(\omega)\| \geq 4$ in each of the following cases:

(a) $\left\|\Phi\left(\alpha_{1}\right)\right\| \geq 2$ and $\left\|\Phi\left(\alpha_{2}\right)\right\| \geq 3$;

(b) $\left\|\Phi\left(\alpha_{i}\right)\right\| \geq 2$ for $i=1,2,3$.

Lemma 4.4. Let $P$ be a peak of $M$ in $\mathscr{L}_{i}(D)$ and suppose $\|\left(\partial P \cup \partial \mathscr{L}_{i-1}(D)\right) \cap$ $\partial M) \|=2$. If $|P|>1$ then $P=\left\langle D_{1}, D_{2}\right\rangle$ such that $a\left(D_{1}\right)+a\left(D_{2}\right)=1$.

Proof. Suppose $|P| \geq 3, P=\left\langle D_{1}, \ldots, D_{k}\right\rangle, k \geq 3$. Consider the extremal regions $D_{1}$ and $D_{k}$. Start with $D_{1}$.

If $a\left(D_{1}\right)=a\left(D_{2}\right)=1$ let $\left\{E_{1}\right\}=\mathcal{A}\left(D_{1}\right)$ and $\left\{E_{2}\right\}=\mathcal{A}\left(D_{2}\right)$. If $E_{1}=E_{2}$ then $v:=\partial D_{1} \cap \partial D_{2} \cap \partial E_{1}$ is an inner vertex with valency three, violating the condition $T(4)$. Hence $E_{1} \neq E_{2}$ and since $D_{1}$ is extremal in $P$ and $D_{2}$ is the only region of $P$ adjacent to $D_{1}$, hence $\mathcal{C}\left(E_{1}\right)=\left\{D_{1}\right\}$ and $d_{M}\left(E_{1}\right)=a\left(E_{1}\right)+b\left(E_{1}\right)+$ $c\left(E_{1}\right) \leq 2+1+1=4$. Consequently, due to the $C(6)$ condition $\partial E_{1} \cap \partial M$ is the product of at least two pieces, hence if $u:=\partial D_{1} \cap \partial E_{1} \cap \partial M$ then $u$ is a vertex with valency three and every piece on $\partial E_{1}$ starting at $u$ and read anticlockwise is contained in $\partial E_{1} \cap \partial M$. Therefore noticing that $d_{M}\left(D_{1}\right)=2$, we may apply Proposition 2.12 to $D_{1}$ to get that 
(i) If $a\left(D_{1}\right)=a\left(D_{2}\right)=1$, then

$$
\left\|\left(\partial E_{1} \cap \partial M\right) \cup\left(\partial D_{1} \cap \partial M\right)\right\| \geq 2 .
$$

(ii) If $a\left(D_{1}\right)=0$ and $a\left(D_{2}\right)=1$, then $d\left(D_{1}\right)=1$, hence by Lemma 2.10

$$
\left\|\left(\partial D_{1} \cap \partial M\right)\right\| \geq 2 .
$$

(iii) If $a\left(D_{1}\right)=1$ and $a\left(D_{2}\right)=0$, then $d\left(D_{1}\right)=2$ and $d\left(D_{2}\right) \leq 2$, hence by Proposition 2.12

$$
\left.\|\left(\partial D_{1} \cap \partial M\right) \cup\left(\partial D_{2} \cap \partial M\right)\right) \| \geq 2 .
$$

It follows from (4.1), (4.2) and (4.3) that if we define $L=\left\langle E_{1}, D_{1}, D_{2}\right\rangle$ if $a\left(D_{1}\right)=1$ and define $L=\left\langle D_{1}, D_{2}\right\rangle$ if $a\left(D_{1}\right)=0$, then $\|\partial L \cap \partial M\| \geq 2$. A similar analysis shows that if $K=\left\langle E_{k}, D_{k}, D_{k-1}\right\rangle$ if $\mathcal{A}\left(D_{k}\right)=\left\{E_{k}\right\}$ and $K=\left\langle D_{k}, D_{k-1}\right\rangle$ if $a\left(D_{k}\right)=0$, then $\|\partial K \cap \partial M\| \geq 2$. Consequently, if $k \geq 4$ then

$$
\left.\|\left(\partial P \cup \partial \mathscr{L}_{i-1}(D)\right) \cap \partial M\right) \| \geq 3,
$$

violating our supposition. Hence $k \leq 3$. Since by assumption $k \geq 3$, we get $k=3$. If one of cases (i) or (ii) above hold for $D_{1}$ (or for $D_{3}$ ) then (4.4) holds true. Assume therefore that case (iii) holds for both $D_{1}$ and $D_{3}$. Then $a\left(D_{1}\right)=1, a\left(D_{2}\right)=0$ and $a\left(D_{3}\right)=1$. Now, $d\left(D_{2}\right)=a\left(D_{2}\right)+b\left(D_{2}\right)+c\left(D_{2}\right)=2+1+0=3$, hence due to the $C(6)$ condition:

$$
\partial D_{2} \cap \partial M \text { is the product of at least three }(6-3=3) \text { pieces }
$$

Since $d\left(D_{1}\right)=d\left(D_{3}\right)=2$, we may apply Proposition 2.12 to the pairs $\left(D_{1}, D_{2}\right)$ and $\left(D_{2}, D_{3}\right)$, where in the notation of Proposition 2.12 in the first pair $D=D_{1}$ and $E_{r}=D_{2}$ while in the second pair $D=D_{3}$ and $E_{\ell}=D_{2}$. By their definition $Q_{\rho}$ and $Q_{\lambda}$ are pieces. Since $E_{\ell}=E_{r}=D_{2}, Q_{\rho}$ is an initial subword of $\Phi\left(\partial D_{2} \cap \partial M\right)$, which is a piece and $Q_{\lambda}$ is a terminal subword of $\Phi\left(\partial D_{2} \cap \partial M\right)$, which is a piece. Since $\left(\partial D_{2} \cap \partial M\right)$ is the product of at least three pieces by (4.5), $Q_{\rho}$ and $Q_{\lambda}$ do not overlap and hence $\|\partial P \cap \partial M\| \geq 3$ violating our supposition. Therefore $|P|=2$ and if $a\left(D_{1}\right)=a\left(D_{2}\right)=0$ or $a\left(D_{1}\right)=a\left(D_{2}\right)=1$ then Lemma 2.10 in the first case and Proposition 2.12 in the second case with the arguments in (i) above imply that $\left\|\left(\partial \mathscr{L}_{i-1} \cup \partial \mathscr{L}_{i}\right) \cap \partial M\right\| \geq 3$. Therefore, $a\left(D_{1}\right)+a\left(D_{2}\right)=1$.

The lemma is proved.

Lemma 4.5. Let $\Lambda$ be a layer decomposition for $M$ and let $P_{1}$ be a peak of $M$ relative to $\Lambda$. If $P_{1}$ is an extremal component of $\mathscr{L}_{i}$ then 
(a) $\left\|\partial P_{1} \cap \partial M\right\| \geq 2$.

(b) Either $P_{1}$ contains a region $D$ with $\|\partial D \cap \partial M\| \geq 2$ or $P_{1}$ contains adjacent regions $D_{1}$ and $D_{2}$ such that $\left\|\left(\partial D_{1} \cup \partial D_{2}\right) \cap \partial M\right\| \geq 2$.

Proof. (a) If $\left|P_{1}\right|=1$ this follows from Lemma 2.10. Assume $\left|P_{1}\right| \geq 2$. Let $P_{1}=\left\langle D_{1}, \ldots, D_{k}\right\rangle, k \geq 2$ and assume $P_{1}$ is left-extremal. Then $b\left(D_{1}\right)=1$ and $c\left(D_{1}\right)=0$. By Theorem 2.7 (c) $a\left(D_{1}\right) \leq 1$. Consequently, $d\left(D_{1}\right) \leq 1+0+1=2$, hence $D_{1}$ is a 2-corner region of $M$. Let $v=\partial D_{1} \cap \partial D_{2} \cap \partial M$. Then by Theorem $2.7(\mathrm{~d}) d_{\mathscr{L}_{i}}(v)=3$ and since $c\left(D_{1}\right)=c\left(D_{2}\right)=0$ hence $d_{\mathscr{L}_{i}}(v)=d_{M}(v)$. Thus $d_{M}(v)=3$ and Proposition 2.12 applies to $D_{1}$. Now, in the notation of Proposition 2.12, $D_{1}=D$ and $D_{2}=E_{r}$ and $d_{M}\left(D_{2}\right)=a\left(D_{2}\right)+b\left(D_{2}\right)+c\left(D_{2}\right) \leq$ $1+2+0=3$, hence $Q_{r}$ is the product of at least three $(6-3=3)$ pieces. (Here, as in Proposition 2.12, $Q_{r}$ is the label of $\partial E_{r} \cap \partial M$.) Therefore, it follows from Proposition 2.12 that $\left\|\left(\partial D_{1} \cap \partial M\right) \cup\left(\partial D_{2} \cap \partial M\right)\right\| \geq 2$, as required. Similarly, if $P_{1}$ is right-extremal then the above argument applies to $D_{k}$.

(b) follows immediate from the proof of part (a).

The lemma is proved.

Now, it follows from Greendlinger's Lemma (see [L-S, p. 250]) that due to the $C$ (4) \& $T$ (4) condition (which is implied by the $C(6) \& T(4)$ condition) $M$ contains at least two $k$-corner regions with $k \leq 2$. Consider the layer structure of $M$ with center $D_{0}$, where $D_{0}$ is a $k$-corner region of $M, k \leq 2$. Since $D_{0}$ is a boundary region of $M$, hence the layer structure of $M$ with center $D_{0}$ has a peak $P_{0}$ in its last layer. Hence by Lemma 4.5 (b) either $P_{0}$ contains a boundary region $D$ such that $\|\partial D \cap \partial M\| \geq 2$ or contains adjacent regions $D$ and $D_{1}$ such that $\left\|\left(\partial D \cup \partial D_{1}\right) \cap \partial M\right\| \geq 2$. Consider the layer structure $\Lambda$ of $M$ with center $D$. Since $d(D) \leq 3$ all the layers of $\Lambda$ are simply connected (i.e. not annular) and in particular its last layer $\mathscr{L}_{p}$ is. If $\mathscr{L}_{p}$ has more than one component then it follows from Lemma 4.5 (a) that $\left\|\partial \mathscr{L}_{p} \cap \partial M\right\| \geq 3$, hence by Lemma $4.3\|\partial M\| \geq 4$, since $\|\partial D \cap \partial M\| \geq 2$ or $\left\|\left(\partial D \cup \partial D_{1}\right) \cap \partial M\right\| \geq 2$, and may assume that $D_{1}, D \nsubseteq \mathscr{L}_{p}$. (If $D_{1} \subseteq \mathscr{L}_{p}$ or $D \subseteq \mathscr{L}_{p}$ then $p \leq 1$ and in this case $\|\partial M\| \geq 4$ easily follows.) Similarly, it follows that

if the interior of $\mathscr{L}_{i}$ contains more than one component then $\|\partial M\| \geq 4$.

Now we turn to the proof of Proposition 4.2.

Proof. First observe that $\|\partial M\| \geq 2$ due to Lemma 4.5 (a) (or Theorem C), and if $\|\partial M\|>2$ then $\|\partial M\| \geq 4$. Suppose by way of contradiction that $M$ is not a onelayer diagram and show that $\|\partial M\| \geq 4$. Let $D, \Lambda$ and $\mathscr{L}_{p}$ be as above. Then due to (4.6) we may assume that $\mathscr{L}_{p}$ has connected interior. It follows that all layers of $\Lambda$ have connected interior.

Let $P=\mathscr{L}_{p}$. Let $\partial M=\alpha \beta$, where $\alpha=\partial D \cap \partial M$ if $\|\partial D \cap \partial M\| \geq 2$ and $\alpha=\left(\partial D \cup \partial D_{1}\right) \cap \partial M$ if $\left\|\left(\partial D \cup \partial D_{1}\right) \cap \partial M\right\| \geq 2$. Then due to Lemmas 4.5 
and 4.3, it is enough to show that $\|\beta\| \geq 3$. Clearly, $\left(\partial P \cup \partial \mathscr{L}_{p-1}\right) \cap \partial M \subseteq \beta$, hence if $\left\|\left(\partial P \cup \partial \mathscr{L}_{p-1}\right) \cap \partial M\right\| \geq 3$ then $\|\partial M\| \geq 4$. Assume therefore that $\|\beta\| \leq 2$ and $\left\|\left(\partial P \cup \partial \mathscr{L}_{p-1}\right) \cap \partial M\right\|=2$. Then by Lemma 4.4 either $|P|=1$ or $P=\left\langle D_{1}, D_{2}\right\rangle$ such that $a\left(D_{1}\right)+a\left(D_{2}\right)=1$.

Claim. Consider the following statement:

$$
\text { either }\left|\mathscr{L}_{i}\right|=1 \text { or } \mathscr{L}_{i}=\left\langle D_{1}, D_{2}\right\rangle \text { such that } a\left(D_{1}\right)+a\left(D_{2}\right)=1 \text {. }
$$

Then $(*)$ holds for every $i, i=1, \ldots, p$.

Proof of the Claim. By the last argument the Claim holds true for $i=p$. Suppose the Claim holds true for $\mathscr{L}_{p}, \ldots, \mathscr{L}_{i}$ and prove for $\mathscr{L}_{i-1}$. Suppose $\left|\mathscr{L}_{i-1}\right| \geq 2$ and let $\mathscr{L}_{i-1}=\left\langle E_{1}, \ldots, E_{k}\right\rangle$. Let $D_{1} \in \mathscr{L}_{i}$ with $a\left(D_{1}\right)=1$ and let $\mathcal{A}\left(D_{1}\right)=\left\{E_{j}\right\}$ for some $j, j=1, \ldots, k$. Assume that either $j \neq 1$ or $j \neq k$. Suppose first $j \neq 1$. If $a\left(E_{1}\right)=0$ then $\left\|\partial E_{1} \cap \partial M\right\| \geq 2$ by Lemma 2.10 , hence $\|\beta\| \geq 3$ by Lemma 4.3, since $\|\partial P \cap \partial M\| \geq 2$ by Proposition 2.13 and $E_{1} \notin P$. This contradicts our assumption that $\|\beta\| \leq 2$. If $a\left(E_{1}\right)=1$ and $a\left(E_{2}\right)=1$ with $\mathcal{A}\left(E_{1}\right)=\left\{F_{1}\right\}$ and $\mathcal{A}\left(E_{2}\right)=\left\{F_{2}\right\}$ then $F_{1} \neq F_{2}$ and $\left\|\left(\partial E_{1} \cup \partial F_{1}\right) \cap \partial M\right\| \geq 2$ hence $\|\beta\| \geq 3$ by Lemma 4.3 (a), contradiction. (See proof of part (i) in Lemma 4.4). Therefore,

(i) if $j \neq 1$ then $a\left(E_{1}\right)=1$ and $a\left(E_{2}\right)=0$.

Suppose now that $j \neq k$. Then the arguments of the case $j \neq 1$ for $E_{k}$ apply and yield

(ii) if $j \neq k$ then $a\left(E_{k}\right)=1$ and $a\left(E_{k-1}\right)=0$.

Assume now that $k \geq 3$. If $j \neq 2$ and $j \neq 1$ then it follows from (i) and Proposition 2.12 that $\left\|\left(\partial E_{1} \cup \partial E_{2}\right) \cap \partial M\right\| \geq 2$ and hence $\mid \beta \| \geq 3$, violating our assumption. Thus

(iii) if $k \geq 3$ then either $j=1$ or $j=2$.

Similarly, if $j \neq k-1$ and $j \neq k$ then it follows from (ii) and Proposition 2.12 that $\left\|\left(\partial E_{k} \cup \partial E_{k-1}\right) \cap \partial M\right\| \geq 2$ and hence $\mid \beta \| \geq 3$, violating our assumption. Thus

(iv) If $k \geq 3$ then either $j=k-1$ or $j=k$.

Therefore, by (iii) and (iv), if $k \geq 3$ then $j \in\{1,2\} \cap\{k-1, k\}$. In particular, $\{1,2\} \cap\{k-1, k\} \neq \emptyset$. It follows that if $k \geq 3$ then $j=k-1=2$, hence $k=3$ and $j=2$. Since $d\left(E_{2}\right)=2$ and $\partial E_{2} \cap \partial D_{1}$ is a piece, either $\partial E_{2} \cap \partial M$ is connected and is the product of at least three $(6-(2+1)=3)$ pieces or $\partial E_{2} \cap \partial M$ has two connected components $\gamma_{1}$ and $\gamma_{3}$ such that $\partial E_{2} \cap \partial S t_{i-1}=\gamma_{1} \gamma_{2} \gamma_{3}$ with $\gamma_{2}=\partial E_{2} \cap \partial D_{1}$ and either $\gamma_{1}$ is the product of at least two pieces or $\gamma_{3}$ is the product of at least two pieces. Therefore we may apply Proposition 2.12 for $E_{1}$ or for $E_{3}$ to give $\left\|\left(\partial \mathscr{L}_{i-1} \cup \partial \mathscr{L}_{i}\right) \cap \partial M\right\| \geq 3$, a contradiction. Consequently, $k \leq 2$, i.e. $\left|\mathscr{L}_{i-1}\right| \leq 2$. 
Now it easily follows by arguments we made several times above that if $\left|\mathscr{L}_{i-1}\right|=2$, then $a\left(E_{1}\right)=1$ and $a\left(E_{2}\right)=1$ would imply that either $\left\|\left(\partial E_{1} \cup \partial F_{1}\right) \cap \partial M\right\| \geq 2$ or $\left\|\left(\partial E_{2} \cup \partial F_{2}\right) \cap \partial M\right\| \geq 2$. This would imply $\|\beta\| \geq 3$, violating our assumption, proving the claim.

We show that $(*)$ implies $M$ is a one-layer diagram. Let $K$ be a region of $M$. Suppose $K$ is in $\mathscr{L}_{i}, 0<i<p$ and $\mathscr{L}_{i}$ consists of two regions. Then $b(K)=1$ by condition $(*)$. Also, $a(K) \leq 1$ by Theorem 2.7 (c), and $c(K) \leq 1$ by condition $(*)$. Hence $d(K) \leq 3$. Let $\mathscr{L}_{i}=\langle K, L\rangle$. If $d(K)=3$ then it follows from (*) that $c(L)=0, a(L)=0$ and $b(L)=1$, hence $d(L)=1$ and hence $\|\partial L \cap \partial M\| \geq 2$ by Lemma 2.10, implying $\|\beta\| \geq 3$. Therefore $d(K) \leq 2$. Suppose $d(K)=1$. Then $\|\partial K \cap \partial M\| \geq 2$ by Lemma 2.10 implying again $\|\beta\| \geq 3$. Therefore $d(K)=2$. Thus, every region in $\mathscr{L}_{i}, 1<i<p$ has exactly two neighbours. But now, $\mathscr{L}_{0}=\{D\}$ and $D$ by $(*)$ has exactly one neighbour (in $\mathscr{L}_{1}$ ) and either $\mathscr{L}_{p}=\left\{D_{1}\right\}$ in which case $d\left(D_{1}\right)=1$ due to the $T(4)$ condition and Theorem $2.7(\mathrm{~d})$, or $\mathscr{L}_{p}=\left\langle D_{1}, D_{2}\right\rangle$ in which case either $d\left(D_{1}\right)=2$ and $d\left(D_{2}\right)=1$ or $d\left(D_{2}\right)=2$ and $d\left(D_{1}\right)=1$, by Lemma 4.4. Consequently, $M$ is a one-layer diagram.

The proposition is proved.

\section{The proofs of Theorems $A$ and $B$}

5.1. Decompositions of $\boldsymbol{R}$. For the proof of Theorem A we may assume $\operatorname{Supp}(R)=$ $I \cup J=\{1,2, \ldots, n\}$. We start with various decompositions of words in $\left\langle G_{I}, G_{J}\right\rangle$. Consider $g p\left\langle G_{I}, G_{J}\right\rangle$ as the amalgamated free product $G_{I} * G_{D} G_{J}$ and denote its length function by $\|\cdot\|$. Every $F$-reduced element $W$ of $G_{I} *_{G_{D}} G_{J}$ with $\|W\| \geq 2$ can be written by

$$
W=W_{1} \ldots W_{k}, \quad k \geq 1,
$$

$F$-reduced as written, such that each of the following holds:

(i) $W_{1} \in \mathcal{T}\left(A_{1} B_{1} K_{1} L_{1}\right), W_{k} \in \mathscr{H}\left(A_{k} B_{k} K_{k} L_{k}\right)$, and $W_{i}=A_{i} B_{i} K_{i} L_{i}$ for $i=2, \ldots, k-1, F$-reduced as written with $A_{i} \in G_{I}, K_{i} \in G_{J}, B_{i} \in G_{D}$ and $L_{i} \in G_{D}, i=1, \ldots, k$;

(ii) $A_{i}$ starts and terminates with an element of $G_{I \backslash D} \backslash\{1\}$;

(iii) $K_{i}$ starts and terminates with an element of $G_{J \backslash D} \backslash\{1\}$.

We call this decomposition of $W$ its $(*)$-decomposition. We say that the $(*)$-decomposition is complete if $W_{1}=A_{1} B_{1} K_{1} L_{1}$ and $W_{k}=A_{k} B_{k} K_{k} L_{k}$. If $W$ is cyclically reduced then it has a cyclic conjugate $W^{*}$ with a complete $(*)$-decomposition.

Since $G_{I}=G_{I \backslash D} * G_{D}$ and $G_{J}=G_{J \backslash D} * G_{D}$, it follows from the normal form theorem for free products (see [L-S, p. 175]) that $W$ has a unique $(*)$-decomposition. As a result, we have the following lemma, the proof of which is a routine application of the normal form theorem for free products, hence we omit it. 
Lemma 5.1. Let $W$ and $S$ be elements of $\left\langle G_{I}, G_{J}\right\rangle$ with (*)-decompositions $W=$ $W_{1} \ldots W_{k}, k \geq 1$ and $S=S_{1} \ldots S_{\ell}, \ell \geq 1$, respectively. Let $W=H P T$ and let $S=H^{\prime} P^{\prime} T^{\prime}$ be decomposition of $W$ and $S$, as words in $F$, reduced as written. Assume that $\|P\| \geq 2$ and $\left\|P^{\prime}\right\| \geq 2$. Then

(a) $P=W_{i}^{\prime \prime} W_{i+1} \ldots W_{j} W_{j+1}^{\prime}$, where $W_{i}^{\prime \prime} \in \mathcal{T}\left(W_{i}\right)$ and $W_{j+1}^{\prime} \in \mathscr{H}\left(W_{j+1}\right)$, reduced as written in $F, S=S_{p}^{\prime \prime} S_{p+1} \ldots S_{q} S_{q+1}^{\prime}$, where $S_{p}^{\prime \prime} \in \mathcal{T}\left(S_{p}\right)$ and $S_{q+1}^{\prime} \in \mathcal{H}\left(S_{q+1}\right)$, reduced as written in $F$.

(b) If $P=P^{\prime}$ and $j \geq i+1$, then $j-i=q-p$ and

(i) $S_{p+t}=W_{i+t}$ for $t=1, \ldots, q-p$;

(ii) $W_{i}^{\prime \prime}=S_{p}^{\prime \prime}$ and $S_{q+1}^{\prime}=W_{j+1}^{\prime}$.

5.2. Word equations that define $\boldsymbol{R}$. Assume now results (i) and (iii) of Theorem A do not hold and consider $H_{I} \cap H_{J}$. We shall prove that necessarily result (ii) holds true. Let $w$ be an element of $H_{I} \cap H_{J}, w \neq 1$. Then there are reduced words $U$ in $G_{I}$ and $V$ in $G_{J}$ such that $w=v(U)=v(V)$. If $U \in G_{D}$ then also $V \in G_{D}$, otherwise $U V^{-1}$ is a non-trivial relation in $G_{J}$, violating Theorem C. Hence if $V \notin G_{D}$ then $U \notin G_{D}$. Since by assumption $H_{D} \neq H_{I} \cap H_{J}$, we may assume $U \in G_{I} \backslash G_{D}$ and $V \in G_{J} \backslash G_{D}$. Now, since $v(U)=v(V)$, we have $v\left(U V^{-1}\right)=1$, hence there is a van Kampen $\mathcal{R}$-diagram with a boundary label $U V^{-1}$. Since $V \notin G_{D}$ and $U \notin G_{D}$, hence $M$ is not-empty. Since we assumed that result (iii) of Theorem A doesn't hold, hence $\left\|R^{*}\right\| \geq 4$ for every cyclic conjugate $R^{*}$ of $R$. Hence Proposition 4.2 applies, implying that $M$ is a one-layer diagram, which without loss of generality has connected interior. Since $\left\|R^{*}\right\| \geq 4$, while $\left\|U V^{-1}\right\|=2$, we get $|M| \geq 2$.

Lemma 5.2. Let $M$ be a connected, simply connected $\mathcal{R}$-diagram with connected interior. Suppose that $M$ is a one-layer diagram; $M=\left\langle D_{0}, \ldots, D_{t}\right\rangle, t \geq 1$, with boundary cycle $u \mu v v^{-1}$ such that $u \in \partial D_{0}$ and $v \in \partial D_{t}$. Suppose $\Phi(\mu) \in G_{I}$ and $\Phi(v) \in G_{J}$. Let $\theta=\partial D_{0} \cap \partial D_{1}$ and let $P=\Phi(\theta)$. Let $\left(P, P^{\prime}\right)=\left(P, P^{\varepsilon}\right)$ be the corresponding piece pair. If $\varepsilon=1$ and $\|R\| \geq 4$ then $R$ has a cyclically reduced (in $F$ ) cyclic conjugate $R^{*}$ with $(*)$-decomposition $W_{1} \ldots W_{k}$, which satisfies the word equation $S W_{j} W_{j+1} Z=Z W_{1} W_{2} S$, where $2 \leq j \leq k-2, S=W_{3} \ldots W_{j-1}$ and $Z=W_{j+1} \ldots W_{k}$.

Proof. Let $\mu_{i}=\mu \cap \partial D_{i}, v_{i}=v \cap \partial D_{i}$, let $H_{i}=\Phi\left(\mu_{i}\right)$ and let $T_{i}=\Phi\left(v_{i}\right)$ for $i=0, \ldots, t$. Consider the subdiagram $\left\langle D_{0}, D_{1}\right\rangle$. (See Figure 7 (a).) Let $\mu=$ $\mu_{0} z_{1} \mu_{1} \ldots z_{t} \mu_{t}$ and $v=v_{0} w_{1} v_{1} \ldots w_{t} v_{t}$, where $z_{i}$ and $w_{i}$ are vertices, $i=1, \ldots, t$. Then $u \mu_{0} z_{1} \theta^{-1} w_{1} v_{0}^{-1}$ is a boundary cycle of $D_{0}$ with label $H_{0} P T_{0}^{-1}$.Now, by Lemma $5.1 R$ has a cyclic conjugate $R^{*}$ with $(*)$-decomposition $R^{*}=W_{1} \ldots W_{k}$ with $W_{i}=A_{i} B_{i} K_{i} L_{i}$ for $i=1,2, \ldots, k, k \geq 2$, like in (i), (ii) and (iii), in the beginning of Section 5.1. We have $k \geq 2$ due to the assumption $\|R\| \geq 4$. 


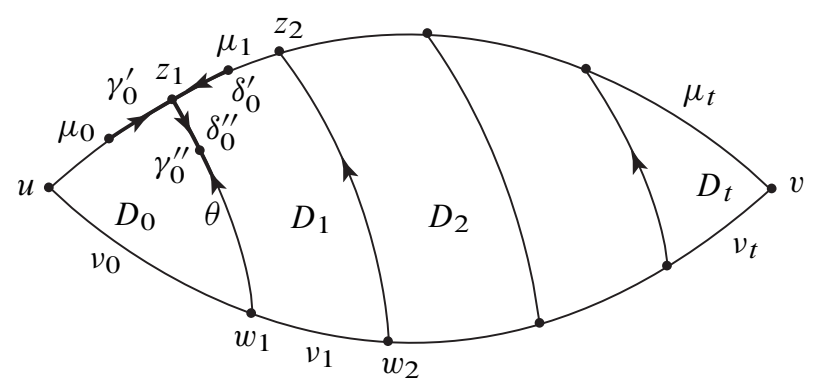

(a)

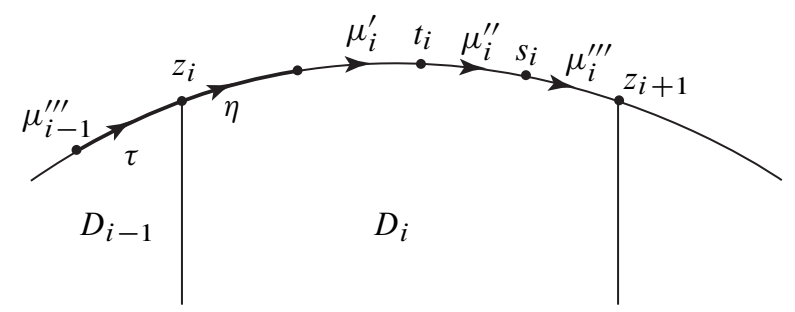

(b)

Figure 7. The diagram for $U=V$.

Since $H_{0} \in G_{I}$ and $T_{0} \in G_{J}, H_{0}$ is a subword of $L_{i} A_{i+1} B_{i+1}$ and $T_{0}^{-1}$ is a subword of $B_{i} K_{i} L_{i}$ for some $i, i=1,2, \ldots, k$. Since $T_{0}^{-1} H_{0}$ is a subword of $R^{*}$, hence $T_{0}^{-1} H_{0}$ is a subword of $B_{i} K_{i} L_{i} A_{i+1} B_{i+1}$. Therefore, $L_{i}$ decomposes to $L_{i}=L_{i}^{\prime} L_{i}^{\prime \prime}$ such that $T_{0}^{-1}$ is a terminal subword of $B_{i} K_{i} L_{i}^{\prime}$ and $H_{0}$ is an initial subword of $L_{i}^{\prime \prime} A_{i+1} B_{i+1}$. Hence,

$P$ has a (*)-decomposition

$$
P=\left(P^{\prime} K_{i+1} L_{i+1}\right) W_{i+2} \ldots W_{k} \ldots W_{i-1}\left(A_{i} P^{\prime \prime}\right),
$$

where $P^{\prime}$ is a terminal subword of $L_{i}^{\prime \prime} A_{i+1} B_{i+1}$ and $P^{\prime \prime}$ is an initial subword of $B_{i} K_{i} L_{i}^{\prime}$.

Now, $P$ is the label of a common boundary path of $D_{0}$ and $D_{1}$, hence $P^{\varepsilon}$ occurs as a subword of $\hat{R}$ (the cyclic word $R$ ) in different positions, for some $\varepsilon \in\{1,-1\}$. (The positions of these occurrences are different because $M$ is a reduced diagram.) Therefore, by Lemma 5.1 either $P$ also has a $(*)$-decomposition

$$
P=\left(Q^{\prime} K_{j+1} L_{j+1}\right) W_{j+2} \ldots W_{j-1}\left(A_{j} Q^{\prime \prime}\right) \text { for somej, }
$$


or a $(*)$-decomposition

$$
P^{-1}=\left(Q^{\prime} K_{j+1} L_{j+1}\right) W_{j+2} \ldots W_{j-1}\left(A_{j} Q^{\prime \prime}\right) \quad \text { for some } j, 1 \leq j \leq n .
$$

Since $\varepsilon=1$ by the assumption of the lemma, $P^{\prime}$ is given by $\left(5.7^{\prime}\right)$. Due to Lemma 5.1 we have

$$
\begin{aligned}
& P^{\prime}=Q^{\prime}, \quad K_{i+1}=K_{j+1}, \quad L_{i+1}=L_{j+1}, \quad W_{i+t}=W_{j+t}, \text { for } t=2, \ldots, k-1, \\
& A_{i}=A_{j} \text { and } P^{\prime \prime}=Q^{\prime \prime}(\text { we count } j+t \text { and } i+t, \bmod k) .
\end{aligned}
$$

Without loss of generality we may assume $i=1$ and $k \geq j>1$. If $k \geq 3$ then $P=$ $\left(P^{\prime} K_{2} L_{2}\right) W_{3} \ldots W_{k}\left(A_{1} P^{\prime \prime}\right)$, hence if $k \geq 3$ we get $W_{3} \ldots W_{k}=W_{j+2} \ldots W_{j-1}$ from (5.9). Denote $[P]=W_{3} \ldots W_{k}, Z=W_{j+2} \ldots W_{k}$ and $S=W_{3} \ldots W_{j-1}$. Since $2 \leq j \leq k$, we have $4 \leq j+2 \leq k+2$. If $j+2 \leq k$ we split $W_{j+2} \ldots W_{j-1}$ into the product $\left(W_{j+2} \ldots W_{k}\right) \cdot\left(W_{1} \ldots W_{j-1}\right)$. Thus

$$
\begin{aligned}
& \text { if } j \leq k-2 \text { then } S W_{j} W_{j+1} Z=Z W_{1} W_{2} S, \\
& \text { where } S=W_{3} \ldots W_{j-1} \text { and } Z=W_{j+1} \ldots W_{k} .
\end{aligned}
$$

If $j+2>k$ then either $j+2=k+1$ or $j+2=k+2$, i.e. either $j=k$ or $j=k-1$.

$$
\text { If } j=k \text { then } W_{2} S=S W_{k} .
$$

Finally, suppose $j=k-1$.

$$
\text { If } j=k-1 \text { then } W_{1} W_{2} S=S W_{k-1} W_{k} .
$$

We claim that cases (5.11) and (5.12) cannot occur. Due to Lemma 5.1 we have in case (5.11) $W_{2}=W_{3}, W_{3}=W_{4}, \ldots, W_{k-1}=W_{k}$, hence $W_{1}=W_{2}=\cdots=W_{k}$. But then $R^{*}=W_{1} \ldots W_{k}=W_{1}^{k}$, a proper power, hence $\mu_{0} \mu_{1}$ is not reduced, a contradiction. In case (5.12) $W_{1}=W_{3}, W_{2}=W_{4}, W_{3}=W_{5}, \ldots, W_{j-2}=W_{k-1}$, $W_{j-1}=W_{k}$. Since $j=k-1$, if $k$ even, we get $W_{1}=W_{2 \ell+1}, \ell=1, \ldots, \frac{k}{2}-1$, $W_{2}=W_{\ell}, \ell=2, \ldots, \frac{k}{2}$. But then $R^{*}=\left(W_{1} W_{2}\right)^{\frac{k}{2}}$ and again $\Phi\left(\mu_{0} \mu_{1}\right)$ is not reduced as written. If $k$ is odd then $W_{1}=W_{2}=\cdots=W_{k}$, i.e. $R^{*}=\left(W_{1}\right)^{k}$, which leads to a contradiction, as above.

The lemma is proved.

In order to find the explicit from of the relator, it is convenient to consider $W_{1}, \ldots, W_{k}$ as symbols, not in $F$ and consider the equation in (5.10) as a word equation in the free semigroup, freely generated by $W_{1}, \ldots, W_{k}$. We can do this due to Lemma 5.1.

We have now to find out the conditions under which the equation in (5.10) is solvable and to find the solutions. To this end we introduce some types of words. 
Definition 5.3 (1-solutions for the defining equations). (a) Let $F_{0}$ be the free group, freely generated by two elements $X_{1}$ and $X_{2}$. For a natural number $\alpha_{0}\left(\alpha_{0} \geq 0\right)$ define: $U_{\alpha_{0}}=\left(X_{1} X_{2}\right)^{\alpha_{0}} X_{1}, V_{\alpha_{0}}=\left(X_{2} X_{1}\right)^{\alpha_{0}} X_{2}, M_{\alpha_{0}}=X_{1}^{\alpha_{0}} X_{2}, N_{\alpha_{0}}=X_{2}^{\alpha_{0}} X_{1}$. (Observe that $U_{\alpha_{0}}, V_{\alpha_{0}}, M_{\alpha_{0}}$ and $N_{\alpha_{0}}$ are in the same orbit under $\operatorname{Aut}\left(F_{0}\right)$.)

(b) Let $k$ be a natural number, $k \geq 1$ and let $\alpha=\left(\alpha_{1}, \ldots, \alpha_{k}\right)$ be a sequence of natural numbers, $\alpha_{i} \geq 0, i=1, \ldots, k$. Define: $U_{\alpha}=U_{\alpha_{1}} \ldots U_{\alpha_{k}}, V_{\alpha}=$ $V_{\alpha_{1}} \ldots V_{\alpha_{k}}, M_{\alpha}=M_{\alpha_{1}} \ldots M_{\alpha_{k}}$, and $N_{\alpha}=N_{\alpha_{1}} \ldots N_{\alpha_{k}}$.

(c) Let $F$ be a free group and let $A$ and $B$ be reduced or empty words in $F$. Let $E$ be the equation $A x y B=B u v A$ over $F$ in the indeterminates $x, y, u$ and $v$. A 1-solution of $E$ is an element $\left(x_{0}, y_{0}, u_{0}, v_{0}\right) \in F^{4}$ with $\left|x_{0}\right|=\left|y_{0}\right|=\left|u_{0}\right|=\left|v_{0}\right|=1$ such that $A x_{0} y_{0} B$ and $B u_{0} v_{0} A$ are reduced as written and $A x_{0} y_{0} B=B u_{0} v_{0} A$ holds true in $F$. $E$ is 1 -solvable over $F$ if it has a 1-solution. Denote $W_{E}=W_{E}\left(u_{0}, v_{0}\right):=$ $B u_{0} v_{0} A$.

Proposition 5.4. Let notation be as in Definition 5.3. Then $E$ is 1-solvable with 1-solution $\left(x_{0}, y_{0}, u_{0}, v_{0}\right)$ if and only if one of the following holds:

I. (i) $W_{E}=u_{0}^{a}, a \geq 0, x_{0}=y_{0}=u_{0}=v_{0}$;

(ii) $W_{E}=\left(u_{0} v_{0}\right)^{a}, a \geq 0, x_{0}=u_{0}$ and $y_{0}=v_{0}$;

(iii) $W_{E}=\left(Y u_{0} v_{0}\right)^{a} Y, B=\left(Y u_{0} v_{0}\right)^{b} Y$, for some non-empty reduced word $Y, a, b \geq 0, x_{0}=u_{0}, y_{0}=v_{0}$

II. Let $\alpha=\left(\alpha_{1}, \ldots, \alpha_{m}\right), m \geq 1,0 \leq \alpha_{i} \in \mathbb{Z}, i=1, \ldots, m$ with the property that if $m \geq 2$ then there exists a natural number $k, 1 \leq k \leq m$ such that one of the following holds:

$$
\left(\alpha_{1}-1, \alpha_{2}, \ldots, \alpha_{k}, \alpha_{k+1}, \ldots, \alpha_{m}\right)=\left(\alpha_{k+1}, \ldots, \alpha_{m}, \alpha_{1}, \ldots, \alpha_{k}-1\right)
$$

or

$$
\left(\alpha_{1}, \ldots, \alpha_{k}, \alpha_{k+1}, \ldots, \alpha_{m}-1\right)=\left(\alpha_{k+1}-1, \ldots, \alpha_{m}, \alpha_{1}, \ldots, \alpha_{k}\right)
$$

Then one of the following holds:

(i) $W_{E}=U_{\alpha}\left(u_{0}, v_{0}\right)$, where $\alpha$ satisfies $(*)$ and $x_{0}=v_{0}, y_{0}=u_{0}$;

(ii) $W_{E}=V_{\alpha}\left(u_{0}, v_{0}\right)$, where $\alpha$ satisfies $(* *)$ and $x_{0}=v_{0}, y_{0}=u_{0}$;

(iii) $W_{E}=M_{\alpha}\left(u_{0}, v_{0}\right)$, where $\alpha$ satisfies $(*)$ and $x_{0}=v_{0}, y_{0}=u_{0}$;

(iv) $W_{E}=N_{\alpha}\left(u_{0}, v_{0}\right)$, where $\alpha$ satisfies $(* *)$ and $x_{0}=v_{0}, y_{0}=u_{0}$;

(v) $W_{E}=\left(Y u_{0}^{2}\right)^{a} Y, a \geq 0, Y$ a reduced word and $x_{0}=y_{0}=u_{0}=v_{0}$.

Proof. The proof of the proposition is straightforward. We first show that for any 1-solution either $x_{0}=u_{0}$ and $y_{0}=v_{0}$, or $x_{0}=v_{0}$ and $y_{0}=u_{0}$. Next we check each of the cases: $A x y B=B y x A$ and $A x y B=B x y A$, respectively. We omit details. 
Definition 5.5 (Exceptional words). (a) Let $W \in F$ be a reduced word and let $V_{1}, V_{2}$ be reduced words. Call $W$ exceptional with respect to $V_{1}, V_{2}$ if $W=W_{E}\left(V_{1}, V_{2}\right)$, where $W_{E}$ is given by one of II(i) - II(iv) of Proposition 5.4. (By $W_{E}\left(V_{1}, V_{2}\right)$ we mean the word obtained from $W_{E}\left(u_{0}, v_{0}\right)$ by substituting $V_{1}$ in place of $u_{0}$ and $V_{2}$ in place of $v_{0}$.)

(b) Let $A$ be a non-empty word in $G_{I}$, reduced in $F$, which starts and terminates with an element from $G_{I \backslash D}$ and let $K$ be a non-empty word in $G_{J}$, reduced in $F$, which starts and terminates with an element from $G_{J \backslash D}$. Let $L, B_{1}$ and $B_{2}$ be elements of $G_{D}, B_{1} \neq B_{2}$ such that $A B_{1} K L$ and $A B_{2} K L$ are reduced in $F$. Define

$$
\widehat{W}_{1}=A B_{1} K L \quad \text { and } \quad \hat{W}_{2}=A B_{2} K L
$$

(c) Let $I, J \subseteq\{1, \ldots, n\}$ be as in the beginning of this section. $W$ is $(I, J)$ exceptional if it is exceptional with respect to $\widehat{W}_{1}$ and $\widehat{W}_{2}$ as given by (5.13).

Thus, $W$ is $(I, J)$-exceptional if $W$ is obtained from $W_{E}$ in parts of II (i) - II (iv) of Proposition 5.4, by substituting $\widehat{W}_{1}$ for $v_{0}$ and $\widehat{W}_{2}$ for $u_{0}$, where $\widehat{W}_{1}$ and $\widehat{W}_{2}$ are given by (5.13).

5.3. The proof of Theorem $A$. We keep the notation and assumptions of Sections 5.1 and 5.2. To simplify notation we shall write $u$ for $u_{0}$ and $v$ for $v_{0}$.

We found in the proof of Lemma 5.2 that in the piece pair $\left(P, P^{\prime}\right)=\left(P, P^{\varepsilon}\right)$, $P$ is given by (5.7), $P^{\prime}$ is given by (5.7 ) if $\varepsilon=1$ and $P^{\prime}$ is given by (5.8) if $\varepsilon=-1$. Assume first $\varepsilon=1$. Then due to Lemma 5.2 we get from (5.10)

$$
\begin{gathered}
R^{*}=W_{1} W_{2}[P], \text { where }[P]=W_{3} \ldots W_{k}=S x y Z=Z u v S \\
W_{j}=x, \quad W_{j+1}=y, \quad W_{1}=u, \quad W_{2}=v, \\
Z=W_{j+1} \ldots W_{k}, \quad S=W_{3} \ldots W_{j-1} \\
R^{*}=u v[P]
\end{gathered}
$$

We apply Proposition 5.4 to (5.3).

We claim that Main Case (I) of Proposition 5.4 and Main Case (II), case (v) cannot occur. Consider first Main Case (I). In this case $x=u$ and $v=y$, hence by (5.3), $W_{j}=W_{1}$ and $W_{j+1}=W_{2}$. The three cases of Main Case (I) are:

(i) $R^{*}=u^{2} u^{a} u^{2} u^{b}=u^{a+b+4}, a, b \geq 0$.

(ii) $R^{*}=(u v)(u v)^{a}(u v)(u v)^{b}=(u v)^{a+b+2}, a+b \geq 1$.

(iii) $R^{*}=(u v)(Y u v)^{a} Y=(u v Y)^{a+1}, a \geq 1$.

Hence in all cases $R^{*}$ is a proper power. Since $M$ is reduced and $u, u v$ and $v$ are not proper powers and $|[P]| \geq 3\|Q\|,(|[P]| \geq 3\|A\|$ in the notation of Lemma 2.6), it follows by Lemma 2.6 that cases (i) and (ii) can not occur. If $u v Y$ is not a proper power 
and $a \geq 2$ or $u v Y=Q^{m}, m \geq 2, Q$ not a proper power, then by Lemma 2.6 these cases cannot occur. Assume therefore that $a=1$ and $u v Y$ is not a proper power. Then $R^{*}=(u v Y)^{2}$. Since $\left\|T_{0}^{-1} H\right\|=2, Y u v Y$ is a subword of $P$, hence $P$ contains $Q(=u v Y)$ as a subword, violating Lemma 2.6. Hence, none of these cases may occur. By a similar argument Case (II) (v) cannot occur. Therefore, by (5.3), $W_{j}=W_{2}$, $W_{j+1}=W_{1}$ and $R^{*}$ is one of the words given by Case (II)(i) -(iv). Consequently, $R^{*}$ is an $(I, J)$-exceptional word, provided that we can show that $A_{1}=A_{2}, K_{1}=K_{2}$ and $L_{1}=L_{2}$. To this end consider $z_{1} \theta^{-1} w_{1}$. (See Figure 7 (a).) Since $H_{0} \in G_{J}, D_{0}$ has a boundary path $\gamma_{0}$ such that $\gamma_{0}=\gamma_{0}^{\prime} z_{1} \gamma_{0}^{\prime \prime}$ with $\Phi\left(\gamma_{0}\right)=W_{1}$ and, similarly, $D_{1}$ has a boundary path $\delta_{0}$ such that $\delta_{0}=\delta_{0}^{\prime} z_{1} \delta_{0}^{\prime \prime}$ with $\Phi\left(\delta_{0}\right)=W_{2}$, and satisfy $\Phi\left(\gamma_{0}^{\prime \prime}\right)=$ $\Phi\left(\delta_{0}^{\prime \prime}\right)$ with $K_{1} L_{1} \in \mathcal{T}\left(\Phi\left(\gamma_{0}^{\prime \prime}\right)\right)$ and $K_{2} L_{2} \in \mathcal{T}\left(\Phi\left(\delta_{0}^{\prime \prime}\right)\right)$. Consequently, $K_{1}=K_{2}$ and $L_{1}=L_{2}$. By a similar argument in $W_{1}$ we get $A_{1}=A_{2}$. Consequently, $W_{1}=A_{1} B_{1} K_{1} L_{1}$ and $W_{2}=A_{1} B_{2} K_{1} L_{1}$. Now $B_{1} \neq B_{2}$ for otherwise $W_{1}=W_{2}$, hence $R^{*}$ is a proper power, which as we saw above can not occur due to Lemma 2.6. Since the last argument applies also for the case $k=2$, it follows that $R^{*}$ is a $(I, J)$-exceptional word, as required.

Next, suppose $\varepsilon=-1$. Then $P^{\prime}$ is given by (5.8). First observe that $W_{j}^{-1}=$ $L_{j}^{-1} K_{j}^{-1} B_{j}^{-1} A_{j}^{-1}$, hence if $W_{j}^{-1}$ is a subword of $W_{1} W_{2}$ then $B_{1}=L_{j}^{-1}, K_{1}=K_{j}^{-1}$, $L_{1}=B_{j}^{-1}$ and $A_{2}=A_{j}^{-1}$. Since $K_{1} \neq K_{1}^{-1}$ and $A_{1} \neq A_{2}^{-1}$, we have $j \notin\{1,2\}$.

Hence, if $k=3$ then $j=3$ and therefore $P^{-1}=Q^{\prime} K_{1} L_{1}\left(A_{2} B_{2} K_{2} L_{2}\right) A_{3} Q^{\prime \prime}$, hence $P=Q^{\prime \prime-1} A_{3}^{-1} L_{2}^{-1} K_{2}^{-1} B_{2}^{-1} A_{2}^{-1} L_{1}^{-1} K_{1}^{-1} Q^{\prime-1}$. On the other hand, by (5.7) $P=P^{\prime} K_{2} L_{2} A_{3} B_{3} K_{3} L_{3} A_{1} P^{\prime \prime}$, where $P^{\prime} \in \mathcal{T}\left(L_{1}^{\prime \prime} A_{2} B_{2}\right)$ and $P^{\prime \prime} \in \mathcal{H}\left(B_{1} K_{1} L_{1}^{\prime}\right)$. Consequently, by Lemma 5.1 either $A_{3}^{-1}=A_{2}, L_{2}^{-1}=B_{2}, K_{2}^{-1}=K_{2}$, a contradiction since $K_{2} \neq K_{2}^{-1}$, or $A_{3}^{-1}=A_{3}$, again a contradiction. Hence $k \neq 3$. Also, a similar argument shows that $k \neq 2$. Therefore we may assume $k \geq 4$. We have by $\left(5.3^{\prime}\right)$ that $R^{*}=W_{1} W_{2}[P]$. Hence, if $\varepsilon=-1$ then we have the following word equation $W_{1} W_{2}[P] W_{1} W_{2}[P]=Q_{1}[P]^{-1} Q_{2}$ in $F$, for some subwords $Q_{1}$ and $Q_{2}$, which define the occurrence of $[P]^{-1}$, where $\left([P],[P]^{-1}\right)$ is a piece pair. Consequently, either $Q_{1}$ is a subword of $W_{1} W_{2}$ in which case $[P]$ and $[P]^{-1}$ overlap and we have:

$$
W_{1} W_{2}[P]=Q_{1}[P]^{-1} Q_{2}^{\prime} \text {, where } Q_{2}^{\prime} \text { is a head of } Q_{2} \text {, or empty, }
$$

or $W_{1} W_{2}$ is a subword of $Q_{1}$ in which case

$$
\begin{array}{r}
{[P]^{-1}=Q_{1}^{\prime \prime} W_{1} W_{2} Q_{1}^{\prime \prime \prime}, \text { where } Q_{1}^{\prime \prime} \in \mathcal{T}([P]), \text { or empty and }} \\
Q_{1}^{\prime \prime \prime} \in \mathscr{H}([P]), \text { or empty. }
\end{array}
$$

Consider equation (5.15). $[P]^{-1}=X c, c \in G_{i}$ for some $i$, by Lemma 2.1 (c) and $W_{1} W_{2}=c^{\prime} X, c^{\prime} \in G_{i}$. Therefore $R^{*}=W_{1} W_{2}[P]=W_{1} W_{2} c W_{2}^{-1} W_{1}^{-1} c^{\prime}$. But then $W_{1} W_{2}$ is a piece and $R^{*}$ is a product of at most three pieces, contradicting the $C(6)$ condition. Finally, consider equation (5.16). We have $[P]=U_{1} Q_{1}^{\prime \prime}$ and 
$[P]=Q_{1}^{\prime \prime \prime} U_{2}$. From equation (5.16) we also have $[P]=\left(Q_{1}^{\prime \prime \prime}\right)^{-1} W_{2}^{-1} W_{1}^{-1}\left(Q_{1}^{\prime \prime}\right)^{-1}$. These three equations imply, due to Lemma 2.1 (c) that $Q_{1}^{\prime \prime}, Q_{1}^{\prime \prime \prime} \in G_{i}$, hence again we get $R^{*}=W_{1} W_{2} c_{1}\left(W_{1} W_{2}\right)^{-1} c_{1}$, contradicting the $C(6)$ condition.

Theorem A is proved.

5.4. The proof of Theorem B. Let notation be as in Figure 7. By Theorem A either $\|R\|=2$ or $\|R\| \geq 4$ and $R^{*}$ has the form

$$
A_{1} B_{i_{1}} K_{1} L_{1} \cdot A_{1} B_{i_{2}} K_{1} L_{1} \ldots A_{1} B_{i_{k+2}} K_{1} L_{1}
$$

where $B_{i_{j}} \in\left\{B_{1}, B_{2}\right\}$. Assume $\|R\| \geq 4$. Then $\Phi\left(\mu_{i}\right)=X_{i} Y_{i} Z_{i}$, where $Y_{i} \in$ $G_{D} *\left\langle A_{1}\right\rangle, X_{i}$ is a terminal subword of a word in $G_{D} *\left\langle A_{1}\right\rangle$ and $Z_{i}$ is an initial subword of a word in $G_{D} *\left\langle A_{1}\right\rangle$. Now, let $z_{i} \mu_{i} z_{i+1}=z_{i} \mu_{i}^{\prime} t_{i} \mu_{i}^{\prime \prime} s_{i} \mu_{i}^{\prime \prime \prime} z_{i+1}$, where $z_{i}, t_{i}, s_{i}, z_{i+1}$ are vertices and $\Phi\left(\mu_{i}^{\prime}\right)=X_{i}, \Phi\left(\mu_{i}^{\prime \prime}\right)=Y_{i}$ and $\Phi\left(\mu_{i}^{\prime \prime \prime}\right)=Z_{i}$. We claim that

if $\Phi\left(\mu_{i}^{\prime}\right)$ has a head $\eta$ such that $\Phi(\eta)$ is in $G_{I}$ then it is a tail of $A_{1}^{ \pm 1}$ and $\mu_{i-1}^{\prime \prime \prime}$ has a tail $\tau$, such that $A_{1}^{ \pm 1}$ is a head of $\Phi\left(\tau z_{i} \mu_{i}^{\prime}\right)$.

Since $A$ is the unique maximal subword of $R^{*}$ in $G_{I}$, which starts and ends with a letter from $G_{I \backslash D}$ this is clear if $d_{M}\left(z_{i}\right)=3$. (See Figure 7 (b).) Now it follows by induction on $d_{M}\left(z_{i}\right)$, by the same argument, that $A_{1}^{ \pm 1}$ is a head of $\Phi\left(\tau z_{i} \mu_{i}^{\prime}\right)$. This proves that $\Phi(\mu) \in G_{D} *\langle A\rangle$. By the same argument $\Phi(v) \in G_{D} *\langle K\rangle$. Also, observe that the above arguments clearly apply for the case $\|R\|=2$.

Theorem B is proved.

\section{References}

[Co] D. J. Collins, Intersections of Magnus subgroups of one-relator roups. In Groups: topological, combinatorial and arithmetic aspects, London Math. Soc. Lecture Note Ser. 311, Cambridge Univ. Press, Cambridge 2004, 255-296. Zbl 1078.20031 MR 2073350

[E-H] M. Edjvet and J. Howie, Intersections of Magnus subgroups and embedding theorems for cyclically presented groups. Preprint 2005.

[Ho1] J. Howie, How to generalize one-relator group theory. In Combinatorial group theory and topology (Alta, Utah, 1984), Ann. of Math. Stud. 111, Princeton University Press, Princeton, NJ 1987, 53-78. Zbl 0632.20021 MR 0895609

[Ho2] J. Howie, Magnus intersections in one-relator products. Michigan Math. J. 53 (2005), 597-623. Zbl 1101.20016 MR MR2207211

[Ju1] A. Juhász, Small cancellation theory with a unified small cancellation condition. I. $J$. London Math. Soc. (2) 40 (1989), 57-80. Zbl 0647.20030 MR 1028914

[Ju2] A. Juhász, Cyclic presentations and solutions of singular equations over free groups. Publ. Math. Debrecen 69 (2006), 333-340. Zbl 05150122 MR 2273985 
[Ju3] A. Juhász, On a Freiheitssatz for cyclic presentations. Internat. J. Algebra Comput. 17 (5-6) (2007), 1043-1053.

[Ju4] A. Juhász, Singular equations with solutions of finite order. Manuscript 2005.

[Ju5] A. Juhász, Solution of the membership problem for Magnus subgroups in certain onerelator free products. In Geometric group theory, Trends Math., Birkhäuser, Basel 2007, 169-195.

[Ju6] A. Juhász, A Freiheitssatz for subsemigroups of one-relator groups with small cancellation condition. In Semigroups and formal languages (Lisbon 2005), World Scientific, Singapore 2007, 111-128.

[Ju7] A. Juhász, Intersection of conjugates of Magnus subgroups in one-relator free groups and free products with small cancellation. In preparation.

[L-S] R. C. Lyndon and P.E. Schupp, Combinatorial group theory. Ergeb. Math. Grenzgeb. 89, Springer-Verlag, Berlin 1977. Zbl 0368.20023 MR 0577064

[MKS] W. Magnus, A. Karrass, and D. Solitar, Combinatorial group theory. 2nd rev. ed., Dover, New York 1976. Zbl 0362.20023 MR 0422434

Received January 29, 2007; revised June 17, 2007

Arye Juhász, Department of Mathematics, Technion - Israel Institute of Technology, 32000

Haifa, Israel

E-mail: arju@techunix.technion.ac.il 